\title{
Guanabenz treatment improves Oculopharyngeal muscular dystrophy phenotype
}

\author{
Alberto Malerba ${ }^{1} *$, Fanny Roth $^{2} *$, Pradeep Harish ${ }^{1}$, Jamila Dhiab $^{2}$, Ngoc Lu-Nguyen ${ }^{1}$, Ornella \\ Cappellari $^{3}$, Susan Jarmin ${ }^{1}$, Alexandrine Mahoudeau ${ }^{2}$, Victor Ythier ${ }^{2}$, Jeanne Lainé ${ }^{2}$, Elisa \\ Negroni ${ }^{2}$, Emmanuelle Abgueguen ${ }^{4}$, Martine Simonelig ${ }^{5}$, Philippe Guedat ${ }^{4}$, Vincent Mouly ${ }^{2}$, \\ Gillian Butler-Browne ${ }^{2}$, Cécile Voisset ${ }^{6}$, George Dickson ${ }^{1 \#}$, Capucine Trollet ${ }^{2 \#}$ \\ ${ }^{1}$ School of Biological Sciences, Centers of Gene and Cell Therapy and Biomedical Sciences, \\ Royal Holloway, University of London, Surrey, UK \\ ${ }^{2}$ Sorbonne Université, INSERM, Association Institut de Myologie, Centre de Recherche en \\ Myologie, UMRS974, 47 bd de l'Hôpital, 75013 Paris, France \\ ${ }^{3}$ Comparative Biomedical Sciences, Royal Veterinary College, London, UK. \\ ${ }^{4}$ Inflectis Bioscience, Nantes, France. \\ ${ }^{5}$ Institute of Human Genetics, CNRS UMR9002-University of Montpellier, mRNA Regulation \\ and Development, 34396-Montpellier, France \\ 6 UMR1078 "Genetic, Functional Genomic and Biotechnologies", INSERM, EFS, Brest \\ University, IBSAM, Brest, France.
}

Authors marked with * equally contributed to the manuscript as first authors.

Authors marked with \# equally contributed to the manuscript as last authors. 


\section{Correspondence should be sent to:}

Capucine Trollet

Myology Research Center, UMRS 974 UPMC - INSERM - AIM

47, bld de l'hôpital - G.H. Pitié-Salpétrière - Bâtiment Babinski

75651 Paris cedex 13

France

Tel: 33-(0)1 42165715

Fax: 33-(0)1 42165700

Email: capucine.trollet@upmc.fr

And

George Dickson,

School of Biological Sciences,

Royal Holloway, University of London, Egham, Surrey, TW20 0EX, UK.

Tel: 44-(0)1784-443545

Fax: 44-(0)1784 414224

Email: g.dickson@ rhul.ac.uk 


\begin{abstract}
Oculopharyngeal muscular dystrophy (OPMD) is a rare late onset genetic disease affecting most profoundly eyelid and pharyngeal muscles, leading respectively to ptosis and dysphagia, and proximal limb muscles at later stages. A short abnormal (GCG) triplet expansion in the polyAbinding protein nuclear 1 (PABPN1) gene leads to PABPN1-containing aggregates in the muscles of OPMD patients. It is commonly accepted that aggregates themselves, the aggregation process and/or the early oligomeric species of PABPN1 are toxic in OPMD. Decreasing PABPN1 aggregate load in animal models of OPMD ameliorates the muscle phenotype. In order to identify a potential therapeutic molecule that would prevent and reduce aggregates, we tested guanabenz acetate (GA), an FDA-approved antihypertensive drug, in OPMD cells as well as in the A17 OPMD mouse model. We demonstrate that treating mice with GA reduces the size and number of nuclear aggregates, improves muscle force, protects myofibres from the pathology-derived turnover and decreases fibrosis. GA is known to target various cell processes, including the unfolded protein response (UPR), which acts to attenuate endoplasmic reticulum (ER) stress. Here we used a cellular model of OPMD to demonstrate that GA increases both the phosphorylation of the eukaryotic translation initiator factor $2 \alpha$ subunit (eIF2 $\alpha$ ) and the splicing of Xbp1, key components of the UPR. Altogether these data suggest that modulation of protein folding regulation can be beneficial for OPMD and support the further development of guanabenz or its derivatives for treatment of OPMD in humans.
\end{abstract}

Significance Statement: Oculopharyngeal muscular dystrophy (OPMD) is a rare late onset incurable genetic disease characterized by the formation of insoluble aggregates in skeletal muscles. It has been shown that the reduction of aggregates correlates with an improvement of the disease. Here we used a mouse model of OPMD to show that Guanabenz acetate, the active constituent of a marketed but recently discontinued drug for hypertension, decreases the number and the size of aggregates after systemic delivery and improves many aspects of the disease. We also describe experimental evidences explaining the mechanism behind the efficacy of such compound for OPMD. 


\section{Introduction (700 words/ 4500 characters)}

Oculopharyngeal muscular dystrophy (OPMD) is a late onset rare autosomal dominant muscular dystrophy. It affects 1:100000 people in Europe but it is more common in population groups such as the Bukharan Jews (1/600) and in Quebec (1/1000) likely due to founder effects $(1,2)$. OPMD is characterized by progressive eyelid drooping (ptosis), swallowing difficulties (dysphagia) and proximal limb weakness. It is caused by a mutation in the poly $(A)$ binding protein nuclear 1 $(P A B P N 1)$ gene that introduces an abnormal expansion of alanine-encoding (GCN)n trinucleotide repeat in the coding region of exon 1 with 11-18 repeats leading to a mutant protein with an expanded number of alanines (expPABPN1, with 11-18 alanines instead of the normal 10) in the $\mathrm{N}$ terminal domain (3). PABPN1 is involved in several steps of mRNA and non-coding RNA biogenesis: PABPN1 controls mRNA poly(A) tail length $(4,5)$ and regulates the use of alternative polyadenylation (APA) sites $(6,7)$ with a direct effect on mRNA levels and stability. It is involved in splicing regulation $(8,9)$ and in the long non-coding RNA (lncRNAs) (10) and small nucleolar RNA (snoRNA) processing (11). It also has an important role in poly(A)mediated RNA decay or export from the nucleus (4) as well as in RNA hyperadenylation (12). Finally PABPN1 has been shown to be required for paraspeckle formation as well as for RNA editing (13).

PABPN1 expression level varies across mouse and human tissues, and is exceptionally low in skeletal muscle (14). Mutated expPABPN1 is prone to form intranuclear aggregates but only in skeletal muscle $(15,16)$. To date the exact consequence of the presence of these aggregates in muscle still remains poorly understood but recent studies have suggested that expPABPN1 aggregates that also sequesters wild-type PABPN1 in heterozygous patients could itself contribute to phenotypes associated with OPMD by a loss of function mechanism $(13,14,17-19)$. This pathological function of PABPN1 aggregates is supported by the fact that higher frequency of aggregates is observed in severe homozygous patients (20). In addition, reducing PABPN1 aggregation by supplementing drugs such as trehalose or other supposed chaperones has consistently demonstrated enhanced cell survival in cell models of OPMD (21-23) and improved muscle weakness in both mouse and Drosophila models (2, 24, 25). Apoptosis and deregulation of the ubiquitin-proteasome system have also been proposed as potential downstream pathological events triggered by the aggregates $(26,27)$. 
There is currently no cure for OPMD and one of the only option for patients is the surgical cricopharyngeal myotomy to improve swallowing and surgical treatment of ptosis. Some innovative therapies are under development, including a phase I/IIa cell therapy trial involving autologous myoblast transplantation that has shown improvement in the swallowing capacity of OPMD patients with a cell dose effect (28). Several studies have shown promising results in animal models of the disease, mainly in Drosophila and in the A17 mouse model, where expPABPN1 is overexpressed under control of human skeletal muscle actin promoter (HSA1). A gene therapy approach tested in A17 mice showed a strong recovery from the disease (18). As mentioned above, anti-aggregation pharmacological strategies have shown positive results both in vitro and in vivo in the same A17 mouse model of $\operatorname{OPMD}(2,21,25,27)$ and intravenous injection of trehalose is currently being used in a phase II clinical trial (29). Guanabenz acetate (GA), an FDA-approved antihypertensive drug agonist of $\alpha 2$-adrenergic receptors (30), was recently identified to display anti-prion activity through the inhibition of the protein folding activity (PFAR) mediated by the domain V of the large ribosomal RNA, which is a component of the large subunit of the ribosome $(31,32)$. GA was shown to be effective in the Drosophila model of OPMD when provided as oral treatment in the food, where it decreased muscle degeneration and reduced the size of aggregates (24). Deletions of the ribosomal DNA locus (encoding rRNAs mediating PFAR) was also shown to reduce OPMD phenotypes and act synergistically with sub-effective doses of 6-aminophenanthridine, a PFAR inhibitor like GA (24), suggesting a mechanism involving the PFAR $(33,34)$. Furthermore, GA has been shown to play a beneficial role in models of neurological diseases (e.g. Amyotrophic lateral sclerosis) by acting on the unfolded protein response (UPR). It has been proposed that GA could decrease protein burden in the endoplasmic reticulum (ER) and so increase the efficiency of ER chaperones by prolonging protein translation attenuation during ER stress $(35,36)$. ER stress condition activates the UPR through different pathways mediated by Grp78 (also called BiP, immunoglobulin-heavy-chain binding protein): first the dissociation of Grp78 from the protein kinase RNA (PRK)-like ER kinase (PERK) activates PERK that phosphorylates the $\alpha$-subunit of eukaryotic translation initiation factor-2 (eIF2 $\alpha$ ). The phosphorylation of eIF2 $\alpha$ attenuates the Cap-dependent protein translation and induces the translation of protein stress responses such as activating transcription factor 4 (ATF4) or protein phosphatase 1 regulatory subunit 15A (PPP1R15A). This latter has a crucial role in terminating the UPR pathway by binding to the 
catalytic subunit of protein phosphatase 1 holoenzyme (PP1c) and dephosphorylating eIF2 $\alpha$. The second branch of the UPR is initiated by the dissociation of Grp78 from inositol requiring protein $1 \alpha$ (IRE-1 $\alpha$ ), which induces a non-canonical splicing of X-box binding protein 1 (XBP1) mRNA producing the active transcription factor XBP1s that in turn triggers the expression of several genes involved in protein folding. The third pathway is operated by the activating transcription factor 6 (ATF6), a receptor that under ER stress and its dissociation to Grp78 is translocated to the Golgi where it is cleaved to produce a fragment which then acts as a transcription factor to promote expression of $X B P 1 \mathrm{mRNA}$ and other proteins involved in protein folding. It has been proposed that GA binds to PPP1R15A, thus preventing its binding to PP1c $(37,38)$ and prolonging eIF2 $\alpha$ phosphorylation with the effect of reducing the accumulation of misfolded proteins in the ER $(37,39)$, but these data are controversial $(40,41)$.

Here we show that GA supplementation tested in the same dose range used for the marketed hypertensive GA-based drug Wytensin, significantly reduces the number of aggregates, decreases fibrotic collagen deposition and pathological muscle turnover and improves muscle strength in the A17 mouse model of OPMD. As expPABPN1 expression in skeletal muscles induces the expression of ER stress markers, we studied the mechanism underlying the beneficial effects of GA in muscle cells and found that both Ire1 $\alpha$ - and PERK-dependent branches of the UPR are affected by GA administration. Altogether, these results identify GA as a positive molecule for OPMD in the mouse model; they also suggest that ER stress plays a role in OPMD and that the modulation of UPR in response to ER stress has therapeutic potential to attenuate PABPN1 aggregate toxicity.

\section{Results}

\section{Guanabenz effectively reduces PABPN1 aggregates in OPMD muscle cells}

GA has been shown to reduce the size of aggregates in a Drosophila model of OPMD (24). We first tested GA in the OPMD Ala17 muscle cell model. When differentiated, this myogenic cellular model expresses PABPN1-Ala17, resulting in the formation of a large number of nuclear aggregates $(42,43)$. GA was added to the cells at day 2 of differentiation and maintained in the medium for 72 additional hours. In these conditions, treatment with $10 \mu \mathrm{M}$ of GA led to a significant reduction in the number and size of PABPN1-Ala17 aggregates (Fig 1A-C), without 
altering cell differentiation (Figure S1A-D). At lower GA concentrations (ranging from 0.1 to 1 $\mu \mathrm{M}$ ), we observed a dose-dependent reduction in the size of PABPN1-Ala17 aggregates but no effect on their number (Figure S2A-C).

\section{ER stress is detected in skeletal muscle of A17 mice}

GA is known to affect the UPR in response to cellular ER stress $(35-37,44)$. No data are available on the ER stress condition and the relative unfolded protein response in muscles of the A17 mouse model of OPMD. Upon ER stress, it has been shown that the lumen of the ER is enlarged $(45,46)$. Using electron microscopy, we analyzed the skeletal muscle of A17 mice and observed that the muscle ER (sarcoplasmic reticulum, SR) was abnormally expanded in a number of glycolytic fibers suggesting that this cellular organelle is indeed under stress (Figure 2A). In addition, analysis of gene expression profiles of A17 skeletal muscles compared to those of wildtype (WT) mice confirmed that the expression of ER genes (GO term: Endoplasmic reticulum subcompartment) is affected in OPMD mice (Figure 2B). This was further confirmed by qPCR analysis on A17 muscle cDNAs for several crucial UPR-related genes: the chaperones glucoseregulated proteins 78 and 94 (Grp78, Grp94), the DNA damage-inducible transcript 3 Ddit3 (also named Chop), the activating transcription factor 4 (Atf4; downstream of PERK branch) and the DnaJ homolog subfamily C member 3 (Dnajc3; downstream of ATF6 branch) (Figure 2C). These data confirm that expPABPN1 expression in skeletal muscles induces the expression of ER stress markers, indicating that the A17 mouse is a relevant OPMD model to evaluate the effect of GA on ER stress and its therapeutic potential in vivo.

\section{GA treatment induces a favorable change in stress response in Ala17 cells}

GA is known to affect the UPR in response to cellular ER stress by prolonging the phosphorylation of the $\alpha$ subunit of eukaryotic translation initiation factor 2 on $\operatorname{Ser}^{51}$ (P-eIF2 $\alpha$ ), a key component of the PERK branch of UPR, to slow down protein translation, decrease the ER protein accumulation in the ER and so leaving more time for the ER chaperones to correctly fold newly synthetized proteins (37). While no effect was observed in GA-treated versus non-treated control cells, a strong increase in phosphorylated eIF2 $\alpha$ (P-eIF2 $\alpha$ ) was observed in GA-treated Ala17 cells (Figure 3A-B) compared to non-treated Ala17 cells. This effect was also observed at lower doses where phosphorylation was activated for all doses ranging from 10 to $0.05 \mu \mathrm{M}$ of 
GA (data not shown). Significant changes in the expression of the same UPR-related genes were observed in Ala17 treated cells in which Grp78, Grp94, Ddit3, Atf4 and Dnajc3 RNA levels were upregulated compared to non-treated cells, while these levels were unchanged in control treated cells (Figure 3C). As Grp78 also activates the IRE1 $\alpha$ branch of the UPR, we evaluated if Xbpl splicing was affected by GA supplementation. A strong increase in the spliced form of Xbpl (Xbpls) was detected in GA-treated Ala17 cells (Figure 3D) while no effect was observed on GA-treated control cells. The fact that control cells are not perturbed by GA administration indicates that GA itself does not induce UPR, and is consistent with OPMD cells being challenged by ER stress. Whereas in the animal the expression of the mutated form of PABPN1 induces the UPR by itself, in Ala17 cells GA is needed to reveal UPR activation.

Overall these data show that GA treatment in OPMD cells induces a favorable change in stress response, mediated by increased phosphorylation of eIF $2 \alpha$ and Xbp1 splicing activation, which induces UPR-related proteins upregulation. Furthermore, GA treatment only modulates UPR in cells affected by ER stress.

Treatment with GA in the A17 OPMD mouse model efficiently improves muscle strength, decreases pathological aggregates accumulation, myofibre turn-over, and fibrosis

To test the effect of GA administration in the OPMD mouse model, 10-12 week old A17 mice were injected intraperitoneally (i.p.) with $4 \mathrm{mg} / \mathrm{kg}$ of GA $(n=6)$ or with saline $(\mathrm{n}=6)$ three times per week (for a final dose of $12 \mathrm{mg} / \mathrm{kg} /$ week of GA) for 13 weeks. We chose this dose because it is in the range of the dose of drug used for the marketed GA-based Wytensin to treat hypertension in humans (47). Age-matched WT mice (FvB) $(n=6)$ were also injected with saline as controls. At the end of the experiment, all mice gained a similar bodyweights irrespective of the treatment (data not shown). As GA has been shown to induce dizziness, weakness and sedation as possible side-effects (48), locomotor behavior of mice was examined by open field behavioral monitoring both after the first and before the last drug administration in the 13-week long study. After the first injection of GA (or saline in control mice), behavior of mice was monitored for 60 minutes and several parameters describing the animal activity were recorded. This analysis showed that mice treated with GA were less active than mice injected with saline soon after GA administration (One-way ANOVA test, Figure S3A). However, the same analysis performed in mice just before the last injection of the study (with mice that were not under the 
effect of the drug at the time of the analysis) indicated that animals chronically treated with GA for 13 weeks are as active as mice injected with saline (One-way ANOVA test Figure S3B) suggesting no detrimental effect of a prolonged treatment with GA in locomotor activity of these mice. Before harvesting the samples from the treated mice, the forelimb force was analyzed by grip strength analysis. A17 mice have weaker forelimbs strength than FvB WT mice $(25,49)$. The treatment with GA significantly increased the muscle strength of treated A17 mice compared to saline injected A17 mice (Figure 4A). To assess more precisely the muscle strength, TA muscles of saline and GA-treated mice were then analyzed by in situ electrophysiology force measurement. Muscles were stimulated with 9 consecutive trains of impulses from 10 to $180 \mathrm{~Hz}$ and the muscle strength provided after each train of stimulation was measured. GA treatment significantly increased the absolute maximal tetanic force generated by the muscles of A17 mice (Figure 4B-C). Normalization of the maximal tetanic force with the cross-sectional area of the muscles provides a measure called maximal specific force, which is an indication of the muscle strength per unit of the muscle. Administration of GA to A17 mice significantly increased the maximal specific force and normalized the muscle strength to the WT level (Figure 4D). No difference in muscle weight was observed for tibialis anterior (TA), extensor digitorum longus (EDL), soleus (Sol) or gastrocnemius (Gast) (Figure S4). As GA is expected to reduce the amount of aggregates, muscles were analyzed for the presence of PABPN1 aggregates. The treatment with GA significantly reduced both the percentage of nuclei containing aggregates (one-way ANOVA test, from $\sim 31 \%$ to $~ 22 \%$ of nuclei) and the size of aggregates in TA skeletal muscles of A17 mice (Figure 5A-C), while the total level of PABPN1 protein was unchanged (Figure 5D-E). Interestingly the proportion of centrally nucleated myofibres, which is an index of muscle degeneration and regeneration, was reduced by almost $50 \%$ in GA treated TA muscle (from $7.5 \%$ to $4 \%$, Figure 6A) suggesting a reduction on the pathology-derived myofibre turnover. As the OPMD pathology is characterized both in humans and in the A17 mouse model by increased fibrosis and collagen deposition in affected muscles, we stained the FvB and A17 muscles for markers of fibrosis (ie Collagen I, III via Sirius red staining, and Collagen VI via immunostaining). As expected, saline-treated A17 muscles were more fibrotic than age matched saline treated FvB muscles. GA treatment significantly decreased the amounts of collagen proteins detected compared to the wild type levels (Figure 6B-C). Overall these data show that 
GA administration is beneficial in OPMD muscles where it improves muscle strength, and reduces aggregates formation, cell turn-over and fibrosis with no significant side-effects.

\section{Oral administration of GA increases muscle strength and reduces the amount of nuclear aggregates of OPMD mice}

Since the GA-based drug Wytensin has been authorized for oral prescription in tablet form, we tested the effect of GA administered orally in OPMD mice. GA was tested in 10-12 week old A17 mice ( $\mathrm{n}=6$ ) by formulating the drugs in food pellets to provide a final dose of $12 \mathrm{mg} / \mathrm{kg}$ of GA per week. Groups of A17 and FvB male mice ( $n=7$ and 8 respectively) were also fed with standard chow pellets as control. Food was provided ad libitum, weighed and changed twice a week. During the experiment, mice fed with GA-supplemented chow ate $15 \%$ less food than mice provided with standard chow possibly due to the unpleasant taste of the drugs. However, at the end of the experiment all mice had gained a similar amount of weight (Figure S5A-B). As performed for the intraperitoneally-delivered drug experiments, we assessed whether GA affects locomotor activity of the mice and again no difference was observed between control groups and GA-treated mice for any of the parameters analyzed at the end of the treatment period (Figure S6). Analysis of PABPN1 positive aggregates showed a decrease in both the percentage of nuclei containing aggregates and the size of the aggregates in muscles treated with GA compared with muscles of control mice (Figure 7A-C). However we did not observe a change in the percentage of centrally nucleated fibres (data not shown). As for the experiment based on i.p. administration, no change was observed in the muscle weight of TA, EDL, Sol and Gast at the end of the study (data not shown). Forelimb strength showed a strong trend towards an improvement in A17 mice treated with GA compared with control mice (unpaired t-test: $* \mathrm{p}=0.038$ ) and was essentially normalized to the level of WT FvB mice (Figure 7D). Overall these results indicate that GA provided orally is also effective in A17 mice by increasing the muscle strength and significantly reducing the size and number of aggregates. 


\section{Discussion}

OPMD is a late onset disease associated with the formation of intracellular aggregates forming nuclear inclusions in affected tissues, similar to what is found in other adult neurological proteinopathies like Huntington's, Parkinson's, Alzheimer's disease, frontotemporal dementia, spinocerebellar ataxias, inclusion body myositis, spinal and bulbar muscular atrophy, myotonic dystrophy and prion diseases like Creutzfeldt-Jakob (50-52). At present, the contribution of PABPN1 aggregates to the OPMD phenotype is still a subject of debate, but it is commonly accepted that aggregates, the aggregation process and/or the early oligomeric species of expPABPN1 are toxic and crucially involved in the OPMD pathology (2). The development of pharmacological molecules targeting these nuclear aggregates may therefore provide a valid approach for the treatment of OPMD. Several drugs have demonstrated positive effects in preclinical models of OPMD, such as 6-aminophenanthridine (6AP) and GA in Drososphila (24), and doxycyclin (25), cystamine (27) and trehalose in mice, with the latter progressed to clinical trial stages in OPMD patients (NCT02015481 on clinicaltrial.gov). In this study, we tested the effect of GA in a cellular model of OPMD and its therapeutic potential in a relevant mouse model of the disease. First of all, we showed that GA decreases the percentage and size of nuclear aggregates both in vitro and in vivo. GA and 6AP have previously been shown to reduce the size of aggregates and improve the pathology in a Drosophila model of OPMD (24). However, while 6AP was shown to use the ribosome-borne protein folding activity of the large ribosomal RNA (PFAR) mechanism to reduce the size of aggregates, the mechanism of action of GA has not been addressed. Several reports, in the context of other diseases, have shown that GA promotes the UPR to counteract ER stress $(35,36,39)$. It has been proposed that GA binds the regulatory subunit of protein phosphatase 1 (PPP1R15A), thereby preventing the dephosphorylation of eiF $2 \alpha$ and then protein translation recovery following stress (37, 39), although its exact mechanism of action remains controversial $(41,53)$. Delaying protein translation recovery may thus allow the UPR downstream chaperones (e.g. GRP78, GRP94) to have more time to cope with the impaired ER homeostasis. Here we show that GA promotes UPR in models of OPMD by acting through the UPR pathway initiated by PERK. In response to GA supplementation, we observed both an increase in phosphorylation of eiF2 $\alpha$ and an increase in Xbpl splicing. It has been described that Xbpl splicing is regulated by activation of both PERK (through increased expression of Atf4 that is known to enhance Xbpl splicing (54)) and IRE1 $\alpha$ pathways. A cross- 
talk between PERK and IRE1 $\alpha$ has been previously demonstrated for miR-30c-2-3p expression that is activated by PERK/NF- $\mathrm{B}$ and inhibits the IRE1-dependant transcription factor Xbpl (55, 56). Here, instead, we show that GA supplementation in vitro increases expression of Atf4 (mediated by PERK pathway) that is known to enhance Xbpl splicing (54). It has been shown that GA on its own is not able to modulate UPR related genes in unstressed cells (37). We found that cells expressing expPABPN1 did not show a different expression of the markers we studied to assess UPR activation compared to control cells. However, Ala17 cells clearly responded to the treatment with GA by upregulating such markers, suggesting that pre-existing ER stress is present in OPMD cells and that this allows a response to GA treatment. A possible explanation for the lack of detection of these UPR signatures in untreated Ala17 cells is that the expression of expPABPN1 in OPMD myotubes is not long enough to allow detection of UPR response. This is further supported by the observation that in the A17 OPMD mouse model, where expPABPN1 is expressed for a longer time, UPR activation is readily detectable. Although our study strongly suggests a connection, the link between PABPN1 aggregates and ER stress in OPMD remains undefined. OPMD is characterized by mitochondrial, ubiquitin proteasome system (UPS) and apoptosis defects $(26,49,57,58)$, all of which can potentially generate ER stress. Furthermore, protein misfolding in the ER, nutrient deprivation, oxidative stress, calcium disturbance, shortage of chaperone proteins and ageing can also trigger ER stress (Integrated stress response, ISR) (5963) and all these features are likely present in OPMD cells. For example it is possible that unresolved ER stress generates more substrates for the UPS that would consequently lead to the overwhelming and saturation of the UPS degradation machinery in a self-reinforcing pathological cycle (64). Alternatively trapping protein chaperones like HSP70 in the aggregates $(65,66)$ would make them less available for normal cellular functions, which could lead to ER stress. Recently, it has been demonstrated that PABPN1 protein regulates nuclear structure and homeostasis of $\mathrm{Ca}^{2+}(67)$. The ER is an essential intracellular organelle for many cell functions including the maintenance of $\mathrm{Ca}^{2+}$ homeostasis. Calcium is one of the key regulators of cell survival and can induce ER stress-mediated apoptosis $(68,69)$ mainly through mitochondrial cell death (70). It has been shown that PABPN1 mutation in OPMD gives rise to multiple $\mathrm{Ca}^{2+}$ defects such as alterations of the sarcoplasmic reticulum (SR) $\mathrm{Ca}^{2+}$ content, inhibition of the voltage-gated SR calcium release and reduced expression of ryanodine receptor (67). Moreover, the presence of TNNT3 pre-mRNA in nuclear aggregates (42) is associated with aberrant calcium 
sensitivity in muscle fibres. These studies show that disturbance of $\mathrm{Ca}^{2+}$ homeostasis plays a role in OPMD physiopathology and suggests that this could be one of the major causes of ER stress in OPMD. Furthermore OPMD has been proposed as a premature-ageing disease (17) and ER stress is prone to be activated in aging since age-related changes such as increased oxidative stress, protein misfolding, impairment of calcium homeostasis and disturbance of protein synthesis are observed when the protein quality is altered (71). Similarly, the enrichment analysis of transcriptomic data from the OPMD mouse revealed ER as one of the top-five deregulated cellular components.

Following the positive effect of GA described in the Drosophila model of OPMD, this study is the first demonstration of the effectiveness of GA in a mammalian model of OPMD. Here, we decided to dose the mice with $12 \mathrm{mg} / \mathrm{kg} /$ week as this translates, after conversion for the body surface area, to approximately $10 \mathrm{mg} / \mathrm{day}$ in a $70 \mathrm{~kg}$ human being which is in the mid-range for the administration of Wytensin, a recently discontinued anti-hypertensive drug whose active ingredient is GA (47). GA i.p. treatment efficiently reduced the percentage of nuclei containing aggregates while simultaneously decreasing muscle fibrosis and improving muscle strength. Furthermore by providing the drug via oral feeding, we demonstrated that the drug was still active and able to decrease the percentage of aggregates using the oral route of delivery, which is compatible with clinical application in humans. A direct comparison with trehalose, the only antiaggregate therapeutic strategy that has yet progressed to clinical application for OPMD, is impossible due to the different doses, route of delivery and outcome measures used to study these compounds. However the efficacy on reducing the amount of aggregates and the functional muscle improvement seem to be at least comparable if not substantially better after administration of GA, and with the further advantage that GA is already a well-characterized drug that entered the market although for a different pathological condition. This drug (ie Wytensin) was discontinued mainly due to some side-effects (e.g. dizziness and lethargy) that we also observed in the current study soon after dosing the mice. However, our study demonstrates that the chronic administration with GA, at least at the doses used, does not affect the locomotor activity in the long term. In conclusion, we suggest that GA ameliorates OPMD acting, at least partially, by finely modulating the ER stress response and reducing the aggregate formation. GA and novel GA derivatives thus have significant therapeutic potential for translation into clinical trials for the treatment of OPMD. 
bioRxiv preprint doi: https://doi.org/10.1101/375758; this version posted July 24,2018 . The copyright holder for this preprint (which was not certified by peer review) is the author/funder. All rights reserved. No reuse allowed without permission. 


\section{Materials and methods}

\section{Drug preparation}

For in vitro experiments, Guanabenz acetate (GA) (G-110, Sigma) was prepared in DMSO at a stock concentration of $100 \mathrm{mM}$. Fresh aliquots were used for each new experiment at concentration ranging from 0.1 to $20 \mu \mathrm{M}$. For the intraperitoneus (IP) injection, GA was first prepared in 5\% DMSO and then diluted to the final concentration at $4 \mathrm{mg} / \mathrm{kg}$ of bodyweight with saline ( $\mathrm{NaCl} 0.9 \%$ in water). For the oral feeding experiments, food supplemented with GA was prepared using the 2016.12 based diet provided by Envigo.

\section{Cell culture}

Primary immortalized mouse myoblasts H2KB IM2 (control cells) are derived from conditionally immortalized mice using a temperature-sensitive SV40 large T-antigen (tsA58) transgene (72). Mutated PABPN1 (Ala17) and wild-type PABPN1 (Ala10) stable cell lines have been established from IM2 cells transfected with a plasmid containing either mutated or wild type PABPN1 under a desmin promoter driving myotube-specific expression (43). Cells were cultivated on matrigel coated surface in DMEM supplemented with $20 \%$ of fotal calf serum (Invitrogen), $0.5 \%$ of chick embryo extract (Seralab), $100 \mathrm{U} / \mathrm{ml}$ penicillin-streptomycin antibiotic (Thermofisher) and 10 $\mathrm{U} / \mathrm{ml}$ interferon-gamma (Millipore) at $33^{\circ} \mathrm{C}$ in a humidified $5 \% \mathrm{CO} 2$ air atmosphere. For Ala10 and Ala17 cell lines containing a resistance gene, $500 \mu \mathrm{g} / \mathrm{ml}$ de geneticin (G418; Life Technologies) was added to the media. At $90 \%$ of confluency, cells were differentiated in DMEM supplemented with $10 \%$ horse serum and $1 \%$ penicillin-streptomycin at $37^{\circ} \mathrm{C}$ in a $5 \%$ $\mathrm{CO}_{2}$ humidified atmosphere.

\section{PABPN1 immunostaining in cells:}

For immunostaining, $\mathrm{H} 2 \mathrm{~KB}$ cells were cultivated in Ibidi $35 \mathrm{~mm}$ Dishes (Biovalley). Immunostaining of PABPN1 was performed at 5 days of differentiation. Cells were washed once in PBS and fixed for 10 min with paraformaldehyde-PBS. Cells were then incubated for 15 min in PBS-glycine 0.1 M and permeabilized in blocking buffer containing 3\% BSA, 5\% goat serum in $0.2 \%$ PBS-Triton X-100. Anti-PABPN1 primary antibody (1/200; ab75855 Abcam) was incubated 1 hour at room temperature in blocking buffer. Cells were washed three times with 
0.1\% PBS-Triton X-100 prior to incubation with goat anti rabbit secondary antibody conjugated to Alexa Fluor 488 (Life Technologies) together with Phalloidin 555 (Interchim).

\section{Western blot}

Proteins were extracted by sonicating the cells in RIPA buffer $(0.15 \mathrm{M} \mathrm{NaCl}, 0.1 \%$ SDS, $50 \mathrm{mM}$ Tris-HCl (pH 8), 2 mM EDTA and 10\% Triton-X-100 with protease inhibitor cocktail (Complete, Roche Diagnostics) and phosphatase inhibitor cocktail (Santa Cruz; sc-45064)). Protein concentration was determined by colorimetric detection method (Pierce BCA Protein Assay; Thermo Fisher). Proteins were separated on 4-12\% Bis-Tris gels (Invitrogen) and transferred onto a PVDF membrane for $1 \mathrm{~h}$ at constant $250 \mathrm{~mA}$ at $4^{\circ} \mathrm{C}$. Membranes were blocked by incubation in $5 \%$ fat free milk or BSA in $1 \mathrm{X}$ TBS, $0.1 \%$ Tween-20 (TBST) for $1 \mathrm{~h}$ at room temperature under agitation. Membranes were stained with primary antibodies (PABPN1 Abcam ab75855; eiF2 $\alpha$-P Cell signaling \#9721; eiF2 $\alpha$ total Cell signaling \#9722) overnight at $4^{\circ} \mathrm{C}$ under agitation. The next day, membranes were washed in TBST and incubated with appropriate secondary antibodies. The G:Box system (Syngene) was used to detect the signals from the membranes.

\section{RT-PCR and Real-time $q R T-P C R$}

Total RNA was extracted from cell and skeletal muscle samples using Trizol reagent (Invitrogen) according to the manufacturer's instructions. RNA quality and purity was determined using a ND-1000 NanoDrop spectrophotometer (NanoDrop Technologies). RNA (100 ng for human muscle biopsies, $1 \mu \mathrm{g}$ for cell pellets and mouse muscle biopsies) was reverse transcribed using M-MLV reverse transcriptase (Invitrogen) according to the manufacturer's instructions. The splicing of Xbpl mRNA was monitored by PCR with $0.5-1 \mu 1$ of cDNA using Reddy mix polymerase (Thermo Scientific) according to the manufacturer's instructions. Primers sparing the $26 \mathrm{bp}$ intron sequences are listed in Table S1. The reaction mixture was heated to $94^{\circ} \mathrm{C}$ for 5 min and followed by 35 PCR cycles: $30 \mathrm{~s}$ at $94^{\circ} \mathrm{C}, 30 \mathrm{~s}$ at $55^{\circ} \mathrm{C}$ and $30 \mathrm{~s}$ at $72^{\circ} \mathrm{C}$ followed by a last elongation step at $72^{\circ} \mathrm{C}$ for $7 \mathrm{~min}$. PCR products from unspliced and alternatively spliced $\mathrm{Xbpl}$ mRNAs, which are 172 and 146 bp, respectively, were resolved on 5\% non-denaturing polyacrylamide or $2 \%$ agarose ethydium bromide stained gels. cDNA was used for quantitative PCR reaction using SYBR green mix buffer (LightCycler 480 Sybr green I Master) in a total 
reaction volume of $9 \mu 1$. The PCR reaction was carried out as follows: 8 min at $95{ }^{\circ} \mathrm{C}$ followed by 50 cycles of the following: $15 \mathrm{~s}$ at $95^{\circ} \mathrm{C}, 15 \mathrm{~s}$ at $60^{\circ} \mathrm{C}$ and $15 \mathrm{~s}$ at $72^{\circ} \mathrm{C}$. Specificity of the PCR products was checked by melting curve analysis using the following program: $65^{\circ} \mathrm{C}$ increasing by $0.11^{\circ} \mathrm{C} / \mathrm{s}$ to $97^{\circ} \mathrm{C}$. The expression level of each mRNA was normalized to that of human $B 2 M$ mRNA (beta-2 microglobulin) or murine RplO mRNA (large ribosomal protein, subunit P0) expression. Expression levels were calculated according to the $\Delta \Delta \mathrm{Ct}$ method. The sequences of primers used for RT-PCR and for real-time qRT-PCR are listed in Table S1.

\section{Mice}

A17 transgenic mice have been previously described $(25,49)$. Male A17 and wild-type FvB (WT) controls were generated by crossing the heterozygous carrier strain A17 with FvB mice. The mice were genotyped by PCR about four weeks after birth. Animals were housed with food and water ad libitum in minimal disease facilities (Royal Holloway, University of London). Ethical and operational permission for in vivo experiments was granted by the RHUL Animal Welfare Committee and the UK Home Office, and this work was conducted under statutory Home Office regulatory, ethics, and licensing procedures, under the Animals (Scientific Procedures) Act 1986 (Project Licence 70/8271).

\section{Drug administration}

For the systemic treatment of OPMD a total of $12 \mathrm{mg} / \mathrm{kg} /$ week of GA was administered using either food chow or intraperitoneus injections. The total amount of GA administered per week is in the range of the dose of the GA based Wytensin used in human (tested in clinical trial from $\sim 0.23$ to $3.7 \mathrm{mg} / \mathrm{kg} /$ week; (73)) for the dose conversion human/mouse see FDA guidance for industry, 2005, Center for Drug Evaluation and Research (CDER), July 2005. For the oral feeding experiment, a 2-week long pilot experiment in FvB healthy mice was conducted to measure the amount of food needed for 13 weeks. The calculation resulted on mice eating an average of 2 pellets (2.8 grams each) per day. For the experiment in OPMD mice, 10-12 week old mice per group (mice fed with GA or bare food) were caged individually and bare or the GAsupplemented pellets (GA was $10 \mathrm{mg} / \mathrm{kg}$ correspondent to $0.028 \mathrm{mg} \mathrm{GA} /$ pellet) changed twice a week for 13 weeks until the end of the experiment. During the experiment, food was weighted each time in order to assess the amount of food eaten by the mice. For the intraperitoneal 
injection, GA was bought from Sigma and diluted in 5\% DMSO in saline. Groups of 10-12 week old A17 or FvB mice were injected with either GA 4 mg/kg/injection or with 5\% DMSO in saline three times per week for 13 weeks.

\section{Assessment of functional readouts in living mice}

\section{Mouse open-field behavioural activity}

Soon after the first i.p. injection of GA or saline, activity of mice was evaluated for 60 minutes (12 bins of 5 minutes each) using open field behavioral monitoring and data obtained per each mouse were averaged. Activity cages from Linton Instruments were used and data were collected using Amonlite software (vs 1.4). During the last 2 weeks of treatment with either oral feeding or intraperitoneal injections, the same cages were used to monitor the activity of treated mice according to a protocol designed following the TREAT-NMD guidelines (TREAT-NMD SOP: DMD_M.2.1.002). Briefly, mice were acclimatized to the test chamber for 60 min every day for 4 consecutive days. On the days of data collection, mice were acclimatized for $30 \mathrm{~min}$ before data acquisition and data were then acquired every 5 minutes for $1 \mathrm{~h}$. This protocol was repeated for 4 days and data obtained per each mouse were averaged. As described in Results, only the first 30 minutes of analyses were considered indicative of the locomotor behavior.

\section{Grip strength test}

The forelimb strength of mice treated with either oral feeding or i.p. injections was assessed using a commercial grip strength monitor (Linton Instrumentation, Norfolk, UK). The protocol described in the TREAT-NMD guidelines was used for the oral feeding experiment ( 5 tests per mouse over a 3-day period, TREAT-NMD SOP: DMD_M.2.2.001). Mice, held from the tail, were allowed to grab a metal mesh attached to a force transducer and the force produced after a gentle pull, was recorded. 30 seconds were left between each of 5 sequential tests per mouse per day. Data were expressed as gram force per gram of the initial body weight. While behavior of the mice orally fed with drugs was normal and similar along the 3-days long procedure, we observed that all mice treated with chronic i.p. injections (and then manipulated thrice/week by the operator) voluntarily did not grab the mesh and preferred to be dragged during both the second and the third days of analyses, so only the measurements obtained from the $1^{\text {st }}$ day of analysis were used for the study. 


\section{In situ muscle physiology}

TA muscle strength was measured by in situ muscle physiology performed under terminal anesthesia using a protocol already reported in (74). Briefly $24 \mathrm{~h}$ after the last injection of saline or GA, muscle function was assessed using tibialis anterior muscles of each mouse. Mice were deeply anesthetized and were carefully monitored throughout the experiment. The distal tendon of the TA muscle was dissected and tied with 4.0 braided surgical silk (Interfocus, Cambridge, UK). The sciatic nerve was exposed and superfluous branches axotomized. The TA tendon was fixed to the lever arm of a 305B dual-mode servomotor transducer (Aurora Scientific, Aurora, Ontario, Canada) via a custom made steel s-hook. Bipolar platinum electrodes located in the distal part of common peroneal nerve, were used to stimulate using supramaximal square-wave pulses of $0.02 \mathrm{~ms}$ (701A stimulator; Aurora Scientific) TA muscles. Data acquisition and control of the servomotors were conducted using a Lab-View-based DMC program (Dynamic muscle control and Data Acquisition; Aurora Scientific). Maximum isometric tetanic force (Po) was determined from the plateau of the force-frequency relationship following a series of stimulations at 10, 30, 40, 50, 80, 100, 120, 150 and $180 \mathrm{~Hz}$. Muscle length was measured using digital calipers based on well-defined anatomical landmarks near the knee and the ankle. The specific force $(\mathrm{N} / \mathrm{cm} 2)$ was calculated by dividing Po by TA muscle cross-sectional area. Overall cross sectional area was estimated using the following formula: muscle weight $(\mathrm{g}) /[\mathrm{TA}$ fiber length $\left.(\mathrm{Lf} ; \mathrm{cm}) \times 0.6 \times 1.06\left(\mathrm{~g} / \mathrm{cm}^{3}\right)\right]$.

\section{Samples harvesting, processing and storing}

Animals were all culled at the end of the study and after the functional analyses. Tibialis anterior (TA), extensor digitorum longus (EDL), soleus (SOL) and gastrocnemius (GAST) muscles were harvested from mice treated with i.p injections and TA, EDL and SOL muscles were collected from mice treated with oral feeding. Muscles from a leg were snap-frozen in liquid nitrogen for WB and RNA studies and the muscles of the contralateral leg were mounted in corks using OCT and frozen in pre-chilled isopentane in liquid nitrogen for histological analyses. All samples were stored at $-80^{\circ} \mathrm{C}$ before analysis.

\section{Immunostaining and histological analyses}


A Bright OTF 5000 cryostat (Bright Instruments, Huntingdon, UK) was used to section muscles at $10 \mu \mathrm{m}$ thickness. Muscle sections, placed on coated glass slides (VWR, Lutterworth, UK) were stored at $-80^{\circ} \mathrm{C}$ prior to use. Immunohistochemical staining was performed using the following antibodies: anti-PABPN1 (rabbit monoclonal, diluted 1:100, Abcam ab75855, ON, $4^{\circ} \mathrm{C}$ ), antiLaminin (rat monoclonal; diluted 1:800; Sigma-Aldrich, 1 h RT), anti-Collagen VI (rabbit monoclonal; diluted 1:400; Abcam ab182744). Alexa fluor (Molecular Probes) antibodies conjugated to 488 or 594 fluorochromes were used to detect the primary antibodies. A published protocol $(18,25,49)$ was used to counterstain the sarcolemma with Laminin for PABPN1 immunostaining. Briefly, after fixing sections in Paraformaldeyde $4 \%$ the slides were incubated with $\mathrm{KCl}$ buffer (1 M KCl, $30 \mathrm{mM}$ HEPES, $65 \mathrm{mM}$ PIPES, $10 \mathrm{mM}$ EDTA, $2 \mathrm{mM} \mathrm{MgCl}$, pH 6.9) for $1 \mathrm{~h}$ at room temperature. Muscle sections were then blocked with $1 \%$ normal goat serum in $0.1 \mathrm{M} \mathrm{PBS}, 0.1 \%$ Triton X100 for $1 \mathrm{~h}$ and then incubated overnight at $4{ }^{\circ} \mathrm{C}$ with both PABPN1 and Laminin primary antibodies diluted in the same buffer. Slides were then washed with $0.1 \%$ tween 20 in PBS and incubated with fluorophore-conjugated secondary antibodies. Slides were mounted with mounting medium Vector containing 4',6-diamidino-2-phenylindole (DAPI, 3 $\mu \mathrm{g} / \mathrm{ml}$; Sigma-Aldrich Ltd.) to detect nuclei. For Collagen VI staining 5\% milk in PBST was used to block for $1 \mathrm{~h}$ at RT the unspecific binding and both the primary and secondary antibodies were incubated for $1 \mathrm{~h}$ at RT in $2.5 \%$ fat free milk in PBST. Five, $20 \mathrm{X}$ magnification images were randomly taken from a blinded observer and used to calculate the percentage of aggregates, the percentage of centrally nucleated fibres and the area covered by collagen VI. A standard protocol for Sirius red was used to detect the collagen I and III in TA muscles. Two to three 10X magnification images were randomly taken from a blinded observer and used to calculate the area stained by Sirius red and expressed as \% on the total area. NIH imageJ analysis software was used to analyze the largest section of each muscle.

\section{Image acquisition and analysis}

Images were visualized using an Olympus BX60 microscope (Olympus Optical, Hamburg, Germany), digitalized using a CCD camera (Photometrics CoolSNAP fx; Roper Scientific) and analyzed using MetaView image analysis system (Universal Imaging, Downington, PA), MetaMorph imaging system (Roper Scientific, Tucson, AZ, USA) software, and ImageJ 1.44o (http://imagej.nih.gov/ij) for quantification analysis. 


\section{Electron microscopy}

TA muscles were dissected, cut into small pieces and immediately fixed in $2 \%$ glutaraldehyde, $2 \%$ paraformaldehyde, $0.1 \mathrm{M}$ phosphate buffer. After abundant washes and $2 \% \mathrm{OsO}_{4}$ postfixation samples were dehydrated at $4^{\circ} \mathrm{C}$ in graded acetone including a $1 \%$ uranyl acetate in $70^{\circ}$ acetone step and were finally embedded in Epon resin. Thin (70nm) sections were stained with uranyl acetate and lead citrate, observed using a Philips CM120 electron microscope (Philips Electronics NV) and photographed with a digital SIS Morada camera.

\section{Gene profile analysis}

Enrichment analysis have been performed on the 1099 differentially expressed genes (DEGs) from quadriceps muscles of 26-weeks old A17 mice previously deposited on GEO (GSE26604) (49). The analysis have been done using overexpressed test (75) of clusterProfiler package (76) and genome wide annotation for Mouse (77) in R. The Benjamini \& Hochberg correction method has been applied and results with a False Discovery Rate (FDR) lower than 5\% and an absolute $\log \mathrm{FC}$ higher than 0.6 have been kept.

\section{Statistical Analysis}

All data are mean values \pm standard error of the mean. Statistical analyses were performed using the Student $t$-test and the one-way ANOVA with the Bonferroni post-hoc analysis as indicated. GraphPad Prism (version 4.0b; GraphPad Software, San Diego California, USA) was used for the analyses. A difference was considered to be significant at $* P<0.05, * * P<0.01$ or $* * * P<0.001$. 


\section{Figures Legend}

\section{Figure 1: GA treatment reduces PABPN1 aggregates in Ala17 differentiated cells.}

(A) Representative immunostaining of PABPN1 aggregates (green) in differentiated Ala17 cells. Nuclei are counterstained with Hoechst (blue) and myotubes are visualized using phalloidin staining (red). Scale bar $=25 \mu \mathrm{m}$. (B) The amount of PABPN1 aggregates was reduced upon GA treatment. Unpaired t-test, $* \mathrm{p}<0.05 \mathrm{n}=3$ experiments with at least 100 nuclei counted per experiment. (C) The size of PABPN1 aggregates is decreased by GA treatment. Cumulative rank plots show the size of nuclear aggregates in A17 GA-treated group compared to A17 untreated group. Kolmogorov-Smirnov test with $* \mathrm{p}<0.05$. NT= cells treated with DMSO only; GA: cells treated with $10 \mu \mathrm{M}$ GA.

Figure 2: ER stress in the OPMD mouse model. (A) Electron micrograph of abnormally enlarged sarcoplasmic reticulum (SR) in A17 mouse. Arrows indicate T-tubules. Scale bar $=2$ $\mu \mathrm{m}$. The boxed area in left panel is enlarged in the right panel. Scale bar $=500 \mathrm{~nm}$. (B) Scatter plot of Cellular component ontology on deregulated genes in A17 muscles compared to FvB muscles. The top 30 cellular components are shown in the bubble chart. Gene ratio is shown on the $\mathrm{x}$-axis, point size shows the gene counts and point color shows the adjusted p-value. (C) Key UPR downstream genes Grp78, Grp94, Ddit3, Dnajc3 and Atf4 are upregulated in A17 mice compared to control mice. 25 week old mice ( $n=6$ per group) were used for this study. Unpaired t-test $* * \mathrm{p}<0.01, * * * \mathrm{p}<0.001, * * * * \mathrm{p}<0.0001$

Figure 3: Both PERK-P-eiF2 $\alpha$ and IRE1-Xbp1 pathways are activated in response to GA treatment in OPMD cells. (A) Western blot showing phosphorylated eiF2 $\alpha$ (P-eiF2 $\alpha$ ) in control and OPMD cells treated with $10 \mu \mathrm{M}$ GA for $72 \mathrm{~h}$ (Ala17 GA) compared to DMSO-treated cells (A17). No increase in phosphorylation of eiF2 $\alpha$ is observed in control cells treated with GA (Ctrl GA). (B) P-eiF2 $\alpha$ normalized to total eiF2 $\alpha$. One-way ANOVA test with Bonferroni post-hoc test, $* * * \mathrm{p}<0,001$. (C) Downstream key UPR markers are overexpressed in Ala17 cells treated with 10 $\mu \mathrm{M}$ GA (red) while unchanged in control cells (green). mRNA levels were normalized to RPLP0 mRNA level (P0). $\mathrm{n}=3$ per group. One-way ANOVA test $* \mathrm{p}<0.05$, *** $\mathrm{p}<0.001$, **** $\mathrm{p}<0.0001$ ns: not significant $(\mathrm{n}=3)$. (D) $10 \mu \mathrm{M}$ GA treatment activates Xbpl alternative splicing in Ala17 
cells but not in control cells, visualized using RT-PCR. The level of total Xbpl is comparable in Ala17 cells and in control cells. Xbpl-u: unspliced form; Xbpl-s: spliced form of Xbpl.

Figure 4: in vivo administration of GA improves the functionality of treated muscles (A) Gripstrength analysis performed in mice at the end of the 13-week long treatment shows that forelimbs of mice treated with GA are significantly stronger than those of mice treated with saline. Unpaired t-test, ${ }^{*} \mathrm{p}<0.05$. (B) in situ muscle physiology in muscles: force-frequency analysis of maximal force generated by TA muscles of treated mice shows that GA administration significantly increases the maximal force generated by TA muscles. One-way Anova test with Bonferroni post-doc test, $* \mathrm{p}<0.05$, $* * * \mathrm{p}<0.001$. (C) Normalization of maximal force by muscle cross sectional area provides a measure of the muscle strength per unit of skeletal muscle called specific maximal force. One-way ANOVA test with Bonferroni post-doc test, $* \mathrm{p}<0.05$, $* * \mathrm{p}<0.01$. (D) Specific maximal force of muscles treated with GA was normalized to the level of control wild type muscles ( $150 \mathrm{hz}$ stimulation is shown). Mean \pm s.e.m., $\mathrm{n}=6$; Oneway ANOVA test with Bonferroni post-doc test, ${ }^{*} \mathrm{p}<0.05$, ${ }^{*} \mathrm{p}<0.01$, ns: not significant. Red and blue stars refer to A17 GA vs A17 saline or FvB saline vs A17 saline comparisons respectively.

Figure 5: Intraperitoneal injection of $G A$ reduces the amount and the size of intranuclear inclusions. After thirteen weeks of treatment by i.p injection of GA, TA muscles of treated mice were collected and PABPN1 expression was analyzed. (A) Detection of PABPN1 intranuclear aggregates (green) and laminin (red) by immunofluorescence in sections of treated muscles. Sections were pre-treated with $1 \mathrm{M} \mathrm{KCl}$ to discard soluble PABPN1 from the tissue. Nuclei are counterstained with DAPI (blue). Scale bar $=200 \mu \mathrm{m}$. (B) Quantification of nuclei containing aggregates in muscle sections indicates that treatment with GA significantly reduces the amount of aggregates to $20 \%$ compared to A17 untreated mice ( 35\% nuclei containing aggregates). One-way ANOVA test with Bonferroni post-hoc test ***p<0.001. (C) GA administration reduces the size of intranuclear aggregates. Cumulative rank plots show the size of aggregates in A17 GA-treated group compared to A17 untreated group. Kolmogorov-Smirnov test with ** p<0.01. (D) Western blot for PABPN1 expression shows that GA treatment does not change the level of PABPN1. (E) Densitometric analysis of western blot for PABPN1 detection shows no difference in PABPN1 expression in saline and GA-treated A17 mice normalized to the relative tubulin 
expression. $\mathrm{n}=6$ in each group. Data are presented as mean \pm s.e.m. One-way ANOVA test with Bonferroni post-hoc test $* * * \mathrm{p}<0.001$, ns, not significant.

Figure 6: GA administration reduces the percentage of centrally nucleated fibres and the fibrosis in muscle. (A) Representative images of histological stainings for Laminin and DAPI were used to quantify the percentage of myofibres with central nuclei. GA treatment significantly reduces the percentage of centrally nucleated fibres $(\mathrm{CN})$ compared to saline treated A17 mice (right panel). One-way ANOVA test with Bonferroni post-hoc test $* * p<0.01, * * * p<0.001$. (B) Representative images of the Sirius red staining used to quantify the area covered by Collagen I and III in TA muscles of treated mice. Muscle of mice treated with GA have significantly less Collagen I and III positive area compared to muscles of mice treated with saline (right panel). One-way ANOVA test with Bonferroni post-hoc test, ***p<0.001, ns, not significant. (C) Representative pictures of Collagen VI immunostaining used to quantify the collagen VI positive (+ve) area. Collagen VI +ve area is smaller in GA-treated muscles compared to saline-treated muscles (right panel) One-way ANOVA test with Bonferroni post-hoc test **p<0.01, $* * * \mathrm{p}<0.001$, ns, not significant. Scale bar $=200 \mu \mathrm{m} . \mathrm{n}=6$ in each group. Data are presented as mean \pm s.e.m.

Figure 7. GA formulated in food has beneficial effects in forelimb strength and reduces the amount and the size of intranuclear aggregates. (A) Detection of PABPN1 nuclear aggregates (green) and laminin (red) by immunofluorescence in sections of treated muscles. Sections were pre-treated with $1 \mathrm{M} \mathrm{KCl}$ to discard all soluble PABPN1 from the tissue. Nuclei are counterstained with DAPI (blue). Scale bar $=200 \mu \mathrm{m}$. (B) Percentage of nuclei containing aggregates in muscle sections indicates that the treatment with GA significantly reduces the amount of aggregates to $20 \%$ compared to A17 untreated mice. One-way ANOVA test with Bonferroni post-hoc test $* * * p<0.001$ (C) GA administration reduces the size of intranuclear aggregates. $n=6$ per group. Cumulative rank plots show the size of aggregates in A17 GA-treated group compared to A17 untreated group. Kolmogorov-Smirnov test with $* * \mathrm{p}<0.01$. (D) Forelimb force of A17 mice treated with GA showed a trend towards an increase in strength compared to the force of mice treated with saline. Unpaired t-test, ${ }^{*} \mathrm{p}<0.05$. ns, not significant. Data are presented as mean \pm s.e.m. $n=6-8$ per group. 


\section{References}

1. Abu-Baker A, Rouleau GA (2007) Oculopharyngeal muscular dystrophy: recent advances in the understanding of the molecular pathogenic mechanisms and treatment strategies. Biochim Biophys Acta 1772(2):173-185.

2. Davies JE (2005) Trehalose reduces aggregate formation and delays pathology in a transgenic mouse model of oculopharyngeal muscular dystrophy. Hum Mol Genet 15(1):23-31.

3. Brais B, et al. (1998) Short GCG expansions in the PABP2 gene cause oculopharyngeal muscular dystrophy. Nat Genet 18(2):164-167.

4. Apponi LH, et al. (2010) Loss of nuclear poly(A)-binding protein 1 causes defects in myogenesis and mRNA biogenesis. Hum Mol Genet 19(6):1058-1065.

5. Benoit B, et al. (2005) An essential cytoplasmic function for the nuclear poly(A) binding protein, PABP2, in poly(A) tail length control and early development in Drosophila. Dev Cell 9(4):511-522.

6. Jenal M, et al. (2012) The poly(A)-binding protein nuclear 1 suppresses alternative cleavage and polyadenylation sites. Cell 149(3):538-553.

7. de Klerk E, et al. (2012) Poly(A) binding protein nuclear 1 levels affect alternative polyadenylation. Nucleic Acids Res 40(18):9089-9101.

8. Bergeron D, Pal G, Beaulieu YB, Chabot B, Bachand F (2015) Regulated Intron Retention and Nuclear Pre-mRNA Decay Contribute to PABPN1 Autoregulation. Mol Cell Biol 35(14):2503-2517.

9. Muniz L, Davidson L, West S (2015) Poly(A) Polymerase and the Nuclear Poly(A) Binding Protein, PABPN1, Coordinate the Splicing and Degradation of a Subset of Human Pre-mRNAs. Mol Cell Biol 35(13):2218-2230.

10. Beaulieu YB, Kleinman CL, Landry-Voyer A-M, Majewski J, Bachand F (2012) Polyadenylation-Dependent Control of Long Noncoding RNA Expression by the Poly(A)Binding Protein Nuclear 1. PLoS Genet 8(11). doi:10.1371/journal.pgen.1003078.

11. Lemay J-F, Lemieux C, St-André O, Bachand F (2010) Crossing the borders: poly(A)binding proteins working on both sides of the fence. RNA Biol 7(3):291-295.

12. Bresson SM, Conrad NK (2013) The Human Nuclear Poly(A)-Binding Protein Promotes RNA Hyperadenylation and Decay. PLoS Genet 9(10). doi:10.1371/journal.pgen.1003893.

13. Banerjee A, Vest KE, Pavlath GK, Corbett AH (2017) Nuclear poly(A) binding protein 1 (PABPN1) and Matrin3 interact in muscle cells and regulate RNA processing. Nucleic Acids Res. doi:10.1093/nar/gkx786.

14. Apponi LH, Corbett AH, Pavlath GK (2013) Control of mRNA stability contributes to low levels of nuclear poly(A) binding protein 1 (PABPN1) in skeletal muscle. Skelet Muscle 3(1):23.

15. Gidaro T, et al. (2013) Atrophy, fibrosis, and increased PAX7-positive cells in pharyngeal muscles of oculopharyngeal muscular dystrophy patients. J Neuropathol Exp Neurol 72(3):234-243.

16. Tomé FM, Fardeau M (1980) Nuclear inclusions in oculopharyngeal dystrophy. Acta Neuropathol (Berl) 49(1):85-87.

17. Anvar SY, et al. (2013) A decline in PABPN1 induces progressive muscle weakness in 
oculopharyngeal muscle dystrophy and in muscle aging. Aging 5(6):412.

18. Malerba A, et al. (2017) PABPN1 gene therapy for oculopharyngeal muscular dystrophy. Nat Commun 8:14848.

19. Vest KE, et al. (2017) Novel mouse models of oculopharyngeal muscular dystrophy (OPMD) reveal early onset mitochondrial defects and suggest loss of PABPN1 may contribute to pathology. Hum Mol Genet 26(17):3235-3252.

20. Blumen SC, et al. (1999) Homozygotes for oculopharyngeal muscular dystrophy have a severe form of the disease. Ann Neurol 46(1):115-118.

21. Abu-Baker A, et al. (2013) Lithium chloride attenuates cell death in oculopharyngeal muscular dystrophy by perturbing Wnt/ $\beta$-catenin pathway. Cell Death Dis 4:e821.

22. Bao YP, Cook LJ, O’Donovan D, Uyama E, Rubinsztein DC (2002) Mammalian, yeast, bacterial, and chemical chaperones reduce aggregate formation and death in a cell model of oculopharyngeal muscular dystrophy. J Biol Chem 277(14):12263-12269.

23. Bao YP, Sarkar S, Uyama E, Rubinsztein DC (2004) Congo red, doxycycline, and HSP70 overexpression reduce aggregate formation and cell death in cell models of oculopharyngeal muscular dystrophy. J Med Genet 41(1):47-51.

24. Barbezier N, et al. (2011) Antiprion drugs 6-aminophenanthridine and guanabenz reduce PABPN1 toxicity and aggregation in oculopharyngeal muscular dystrophy. EMBO Mol Med 3(1):35-49.

25. Davies JE, et al. (2005) Doxycycline attenuates and delays toxicity of the oculopharyngeal muscular dystrophy mutation in transgenic mice. Nat Med 11(6):672677.

26. Anvar SY, et al. (2011) Deregulation of the ubiquitin-proteasome system is the predominant molecular pathology in OPMD animal models and patients. Skelet Muscle 1(1):1.

27. Davies JE, Rose C, Sarkar S, Rubinsztein DC (2010) Cystamine suppresses polyalanine toxicity in a mouse model of oculopharyngeal muscular dystrophy. Sci Transl Med 2(34):34ra40-34ra40.

28. Périé S, et al. (2014) Autologous myoblast transplantation for oculopharyngeal muscular dystrophy: a phase I/IIa clinical study. Mol Ther J Am Soc Gene Ther 22(1):219-225.

29. Argov Z, Gliko-Kabir I, Brais B, Caraco Y, Megiddo D (2016) Intravenous Trehalose Improves Dysphagia and Muscle Function in Oculopharyngeal Muscular Dystrophy (OPMD): Preliminary Results of 24 Weeks Open Label Phase 2 Trial (I4.007). Neurology 86(16 Supplement):I4.007.

30. Nash DT (1973) Clinical trial with guanabenz, a new antihypertensive agent. J Clin Pharmacol 13(10):416-421.

31. Blondel M, et al. (2016) Protein Folding Activity of the Ribosome is involved in Yeast Prion Propagation. Sci Rep 6:32117.

32. Tribouillard-Tanvier D, et al. (2008) Antihypertensive drug guanabenz is active in vivo against both yeast and mammalian prions. PloS One 3(4):e1981.

33. Nguyen PH, et al. (2014) Structure-activity relationship study around guanabenz identifies two derivatives retaining antiprion activity but having lost $\alpha 2$-adrenergic receptor agonistic activity. ACS Chem Neurosci 5(10):1075-1082.

34. Reis SD, et al. (2011) Mode of action of the antiprion drugs 6AP and GA on ribosome assisted protein folding. Biochimie 93(6):1047-1054.

35. Vieira FG, et al. (2015) Guanabenz Treatment Accelerates Disease in a Mutant SOD1 Mouse Model of ALS. PloS One 10(8):e0135570. 
36. Wang L, Popko B, Tixier E, Roos RP (2014) Guanabenz, which enhances the unfolded protein response, ameliorates mutant SOD1-induced amyotrophic lateral sclerosis. Neurobiol Dis 71:317-324.

37. Tsaytler P, Harding HP, Ron D, Bertolotti A (2011) Selective inhibition of a regulatory subunit of protein phosphatase 1 restores proteostasis. Science 332(6025):91-94.

38. Carrara M, Sigurdardottir A, Bertolotti A (2017) Decoding the selectivity of eIF2 $\alpha$ holophosphatases and PPP1R15A inhibitors. Nat Struct Mol Biol 24(9):708-716.

39. Das I, et al. (2015) Preventing proteostasis diseases by selective inhibition of a phosphatase regulatory subunit. Science 348(6231):239-242.

40. Choy MS, et al. (2015) Structural and Functional Analysis of the GADD34:PP1 eIF2 $\alpha$ Phosphatase. Cell Rep 11(12):1885-1891.

41. Crespillo-Casado A, Chambers JE, Fischer PM, Marciniak SJ, Ron D (2017) PPP1R15Amediated dephosphorylation of eIF2 $\alpha$ is unaffected by Sephin1 or Guanabenz. eLife 6. doi:10.7554/eLife.26109.

42. Klein P, et al. (2016) Nuclear poly(A)-binding protein aggregates misplace a pre-mRNA outside of SC35 speckle causing its abnormal splicing. Nucleic Acids Res. doi:10.1093/nar/gkw703.

43. Raz V, et al. (2011) Modeling Oculopharyngeal Muscular Dystrophy in Myotube Cultures Reveals Reduced Accumulation of Soluble Mutant PABPN1 Protein. Am J Pathol 179(4):1988-2000.

44. Neuber C, et al. (2014) Guanabenz Interferes with ER Stress and Exerts Protective Effects in Cardiac Myocytes. PLOS ONE 9(6):e98893.

45. Akiyama M, et al. (2009) Increased insulin demand promotes while pioglitazone prevents pancreatic beta cell apoptosis in Wfs 1 knockout mice. Diabetologia 52(4):653-663.

46. Riggs AC, et al. (2005) Mice conditionally lacking the Wolfram gene in pancreatic islet beta cells exhibit diabetes as a result of enhanced endoplasmic reticulum stress and apoptosis. Diabetologia 48(11):2313-2321.

47. Weidler DJ, Garg DC, Jallad NS (1984) Dose-response relationship of single oral doses of guanabenz in hypertensive patients. J Cardiovasc Pharmacol 6 Suppl 5:S762-765.

48. Walker BR, Deitch MW, Schneider BE, Hare LE, Gold JA (1981) Long-term therapy of hypertension with guanabenz. Clin Ther 4(3):217-228.

49. Trollet C, et al. (2010) Molecular and phenotypic characterization of a mouse model of oculopharyngeal muscular dystrophy reveals severe muscular atrophy restricted to fast glycolytic fibres. Hum Mol Genet 19(11):2191-2207.

50. Lindholm D, Korhonen L, Eriksson O, Kõks S (2017) Recent Insights into the Role of Unfolded Protein Response in ER Stress in Health and Disease. Front Cell Dev Biol 5. doi:10.3389/fcell.2017.00048.

51. Reyes JM, Hoenig EM (1981) Intracellular spiral inclusions in cerebral cell processes in Creutzfeldt-Jakob disease. J Neuropathol Exp Neurol 40(1):1-8.

52. Tanaka S, Saito M, Morimatsu M, Ohama E (2000) Immunohistochemical studies of the PrP(CJD) deposition in Creutzfeldt-Jakob disease. Neuropathol Off J Jpn Soc Neuropathol 20(2):124-133.

53. Crespillo-Casado A, et al. (2018) A Sephin1-insensitive tripartite holophosphatase dephosphorylates translation initiation factor 2 $\alpha$. J Biol Chem 293(20):7766-7776.

54. Tsuru A, Imai Y, Saito M, Kohno K (2016) Novel mechanism of enhancing IRE1 $\alpha$-XBP1 signalling via the PERK-ATF4 pathway. Sci Rep 6:24217.

55. Byrd AE, Aragon IV, Brewer JW (2012) MicroRNA-30c-2* limits expression of 
proadaptive factor XBP1 in the unfolded protein response. J Cell Biol 196(6):689-698.

56. Chhabra R, Dubey R, Saini N (2011) Gene expression profiling indicate role of ER stress in miR-23a 27a 24-2 cluster induced apoptosis in HEK293T cells. RNA Biol 8(4):648664.

57. Chartier A, Benoit B, Simonelig M (2006) A Drosophila model of oculopharyngeal muscular dystrophy reveals intrinsic toxicity of PABPN1. EMBO J 25(10):2253-2262.

58. Chartier A, et al. (2015) Mitochondrial Dysfunction Reveals the Role of mRNA Poly(A) Tail Regulation in Oculopharyngeal Muscular Dystrophy Pathogenesis. PLoS Genet 11(3). doi:10.1371/journal.pgen.1005092.

59. Brown MK, Naidoo N (2012) The endoplasmic reticulum stress response in aging and age-related diseases. Front Physiol 3. doi:10.3389/fphys.2012.00263.

60. Fritz JM, et al. (2014) Deficiency of the BiP cochaperone ERdj4 causes constitutive endoplasmic reticulum stress and metabolic defects. Mol Biol Cell 25(4):431-440.

61. Harding HP, et al. (2003) An Integrated Stress Response Regulates Amino Acid Metabolism and Resistance to Oxidative Stress. Mol Cell 11(3):619-633.

62. Jiang P, Gan M, Lin W-L, Yen S-HC (2014) Nutrient deprivation induces $\alpha$-synuclein aggregation through endoplasmic reticulum stress response and SREBP2 pathway. Front Aging Neurosci 6. doi:10.3389/fnagi.2014.00268.

63. Krebs J, Agellon LB, Michalak M (2015) Ca(2+) homeostasis and endoplasmic reticulum (ER) stress: An integrated view of calcium signaling. Biochem Biophys Res Commun 460(1):114-121.

64. Hamdan N, Kritsiligkou P, Grant CM (2017) ER stress causes widespread protein aggregation and prion formation. J Cell Biol 216(8):2295-2304.

65. Corbeil-Girard L-P, et al. (2005) PABPN1 overexpression leads to upregulation of genes encoding nuclear proteins that are sequestered in oculopharyngeal muscular dystrophy nuclear inclusions. Neurobiol Dis 18(3):551-567.

66. Tavanez JP, et al. (2009) Hsp70 Chaperones and Type I PRMTs Are Sequestered at Intranuclear Inclusions Caused by Polyalanine Expansions in PABPN1. PLoS ONE 4(7). doi:10.1371/journal.pone.0006418.

67. García-Castañeda M, et al. (2017) Functional impact of an oculopharyngeal muscular dystrophy mutation in PABPN1. J Physiol 595(13):4167-4187.

68. Bahar E, Kim H, Yoon H (2016) ER Stress-Mediated Signaling: Action Potential and $\mathrm{Ca}(2+)$ as Key Players. Int J Mol Sci 17(9). doi:10.3390/ijms17091558.

69. Pinton P, Giorgi C, Siviero R, Zecchini E, Rizzuto R (2008) Calcium and apoptosis: ERmitochondria Ca2+ transfer in the control of apoptosis. Oncogene 27(50):6407-6418.

70. Lee K, et al. (2002) IRE1-mediated unconventional mRNA splicing and S2P-mediated ATF6 cleavage merge to regulate XBP1 in signaling the unfolded protein response. Genes Dev 16(4):452-466.

71. Deldicque L (2013) Endoplasmic reticulum stress in human skeletal muscle: any contribution to sarcopenia? Front Physiol 4. doi:10.3389/fphys.2013.00236.

72. Morgan JE, et al. (1994) Myogenic cell lines derived from transgenic mice carrying a thermolabile T antigen: a model system for the derivation of tissue-specific and mutationspecific cell lines. Dev Biol 162(2):486-498.

73. Holmes B, Brogden RN, Heel RC, Speight TM, Avery GS (1983) Guanabenz. A review of its pharmacodynamic properties and therapeutic efficacy in hypertension. Drugs 26(3):212-229.

74. Malerba A, et al. (2011) Chronic systemic therapy with low-dose morpholino oligomers 
ameliorates the pathology and normalizes locomotor behavior in mdx mice. Mol Ther $J$ Am Soc Gene Ther 19(2):345-354.

75. Boyle EI, et al. (2004) GO::TermFinder--open source software for accessing Gene Ontology information and finding significantly enriched Gene Ontology terms associated with a list of genes. Bioinforma Oxf Engl 20(18):3710-3715.

76. Yu G, Wang L-G, Han Y, He Q-Y (2012) clusterProfiler: an R Package for Comparing Biological Themes Among Gene Clusters. OMICS J Integr Biol 16(5):284-287.

77. Carlson M (2018) org.Mm.eg.db: Genome wide annotation for Mouse. R package version 3.6.0. Bioconductor. Available at: http://bioconductor.org/packages/org.Mm.eg.db/ [Accessed June 27, 2018]. 
B
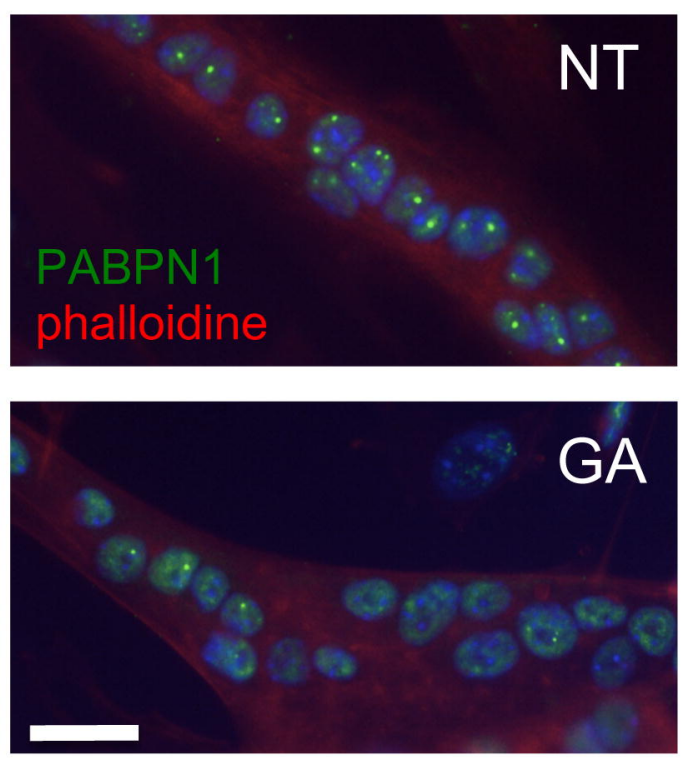

C

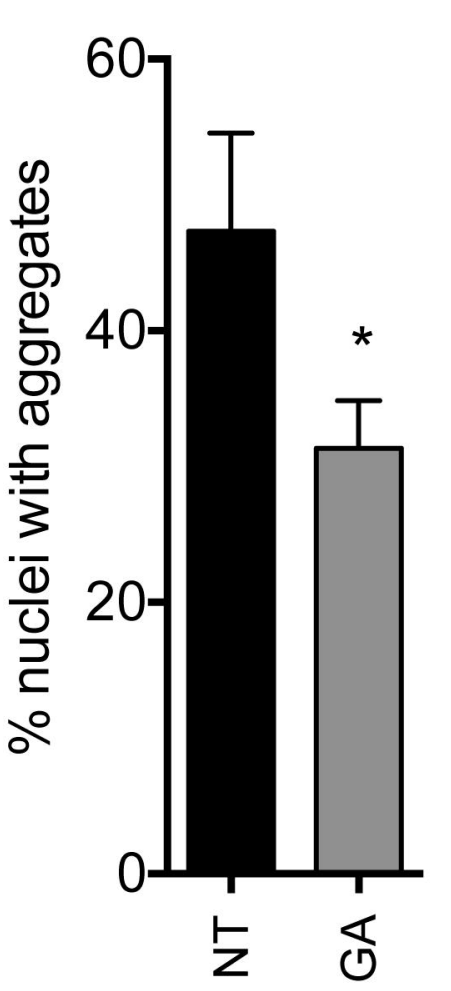

Figure 1

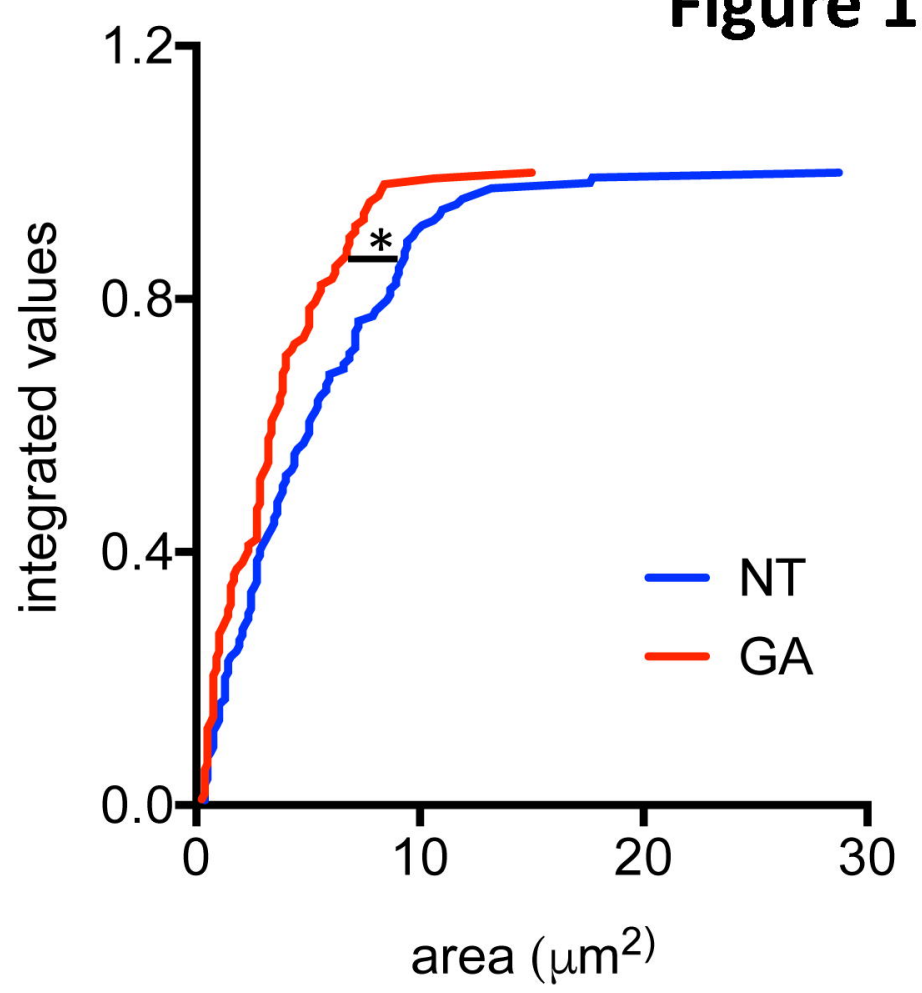




\section{A \\ Ctrl \\ Ala17 \\ Ala17 GA}

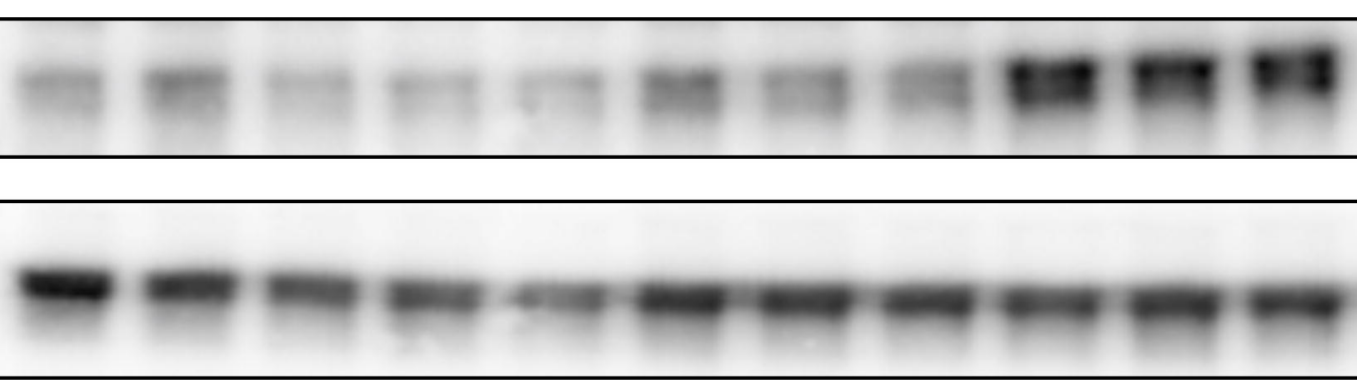

- P-elF2a

- elF2a total

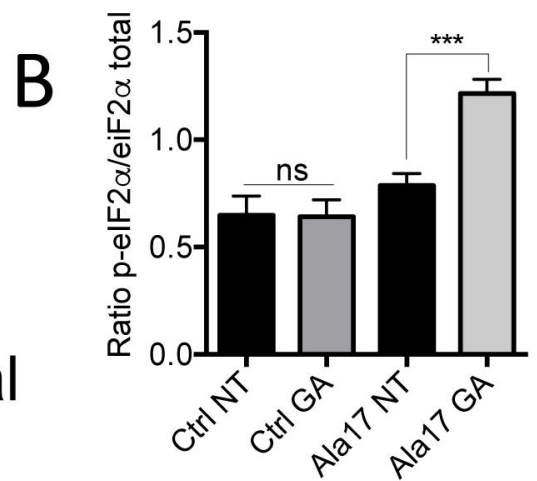

C
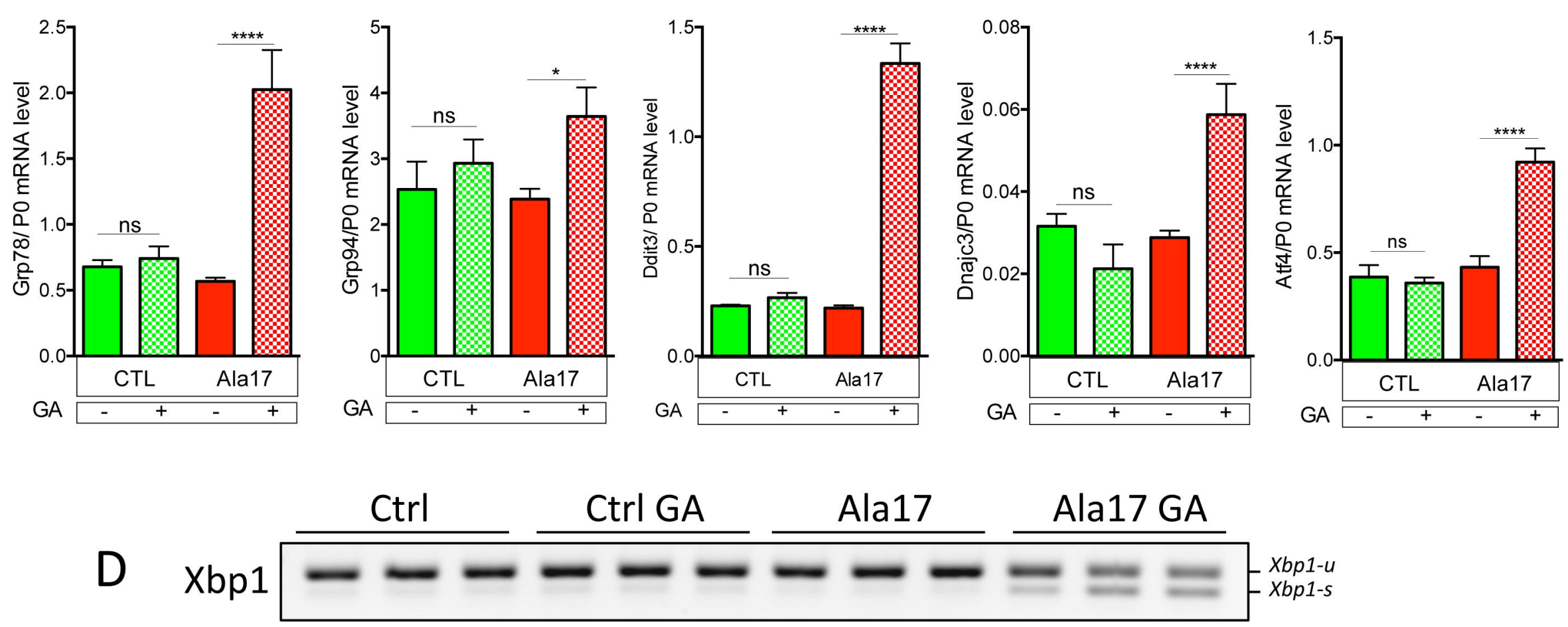

Rplp0

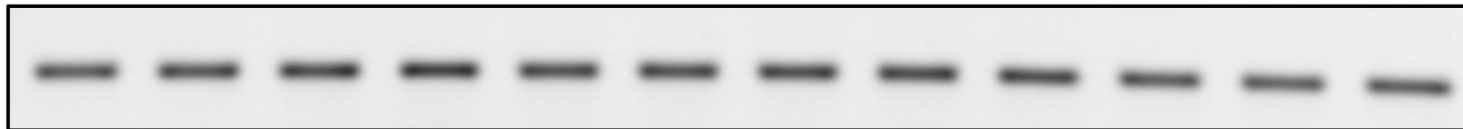

Figure 2 
A
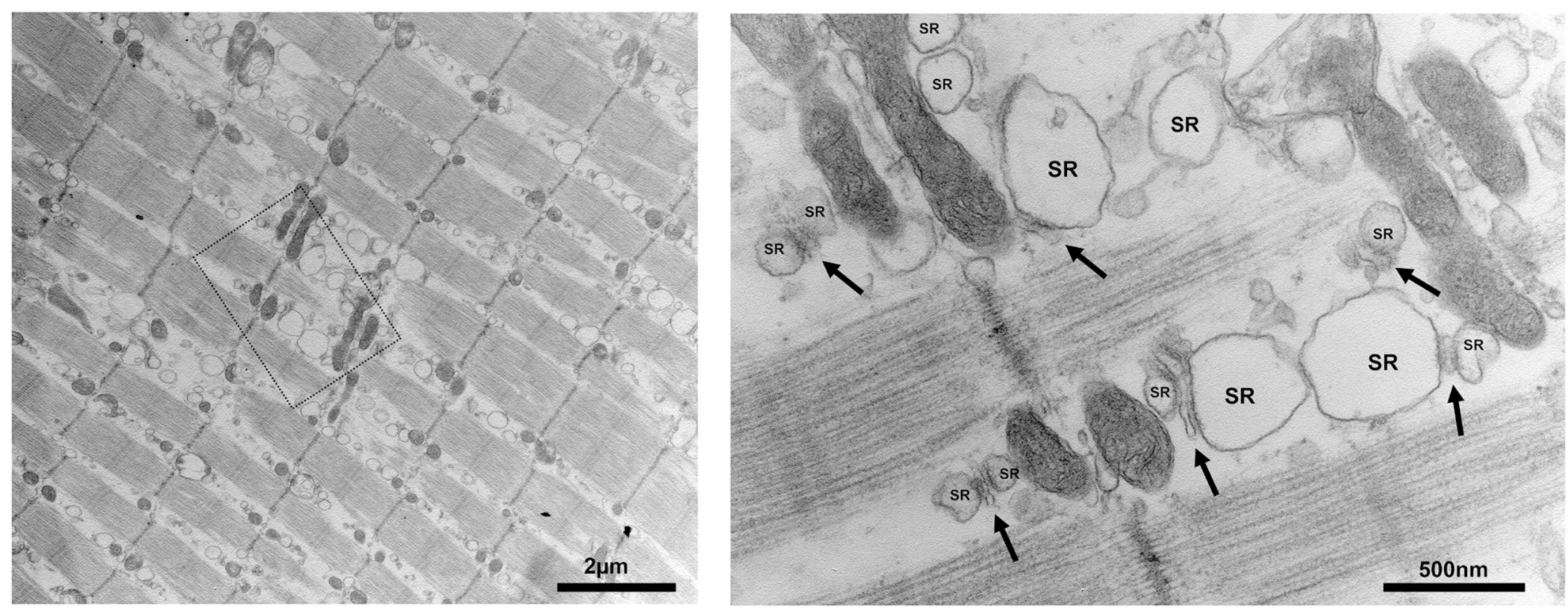

B
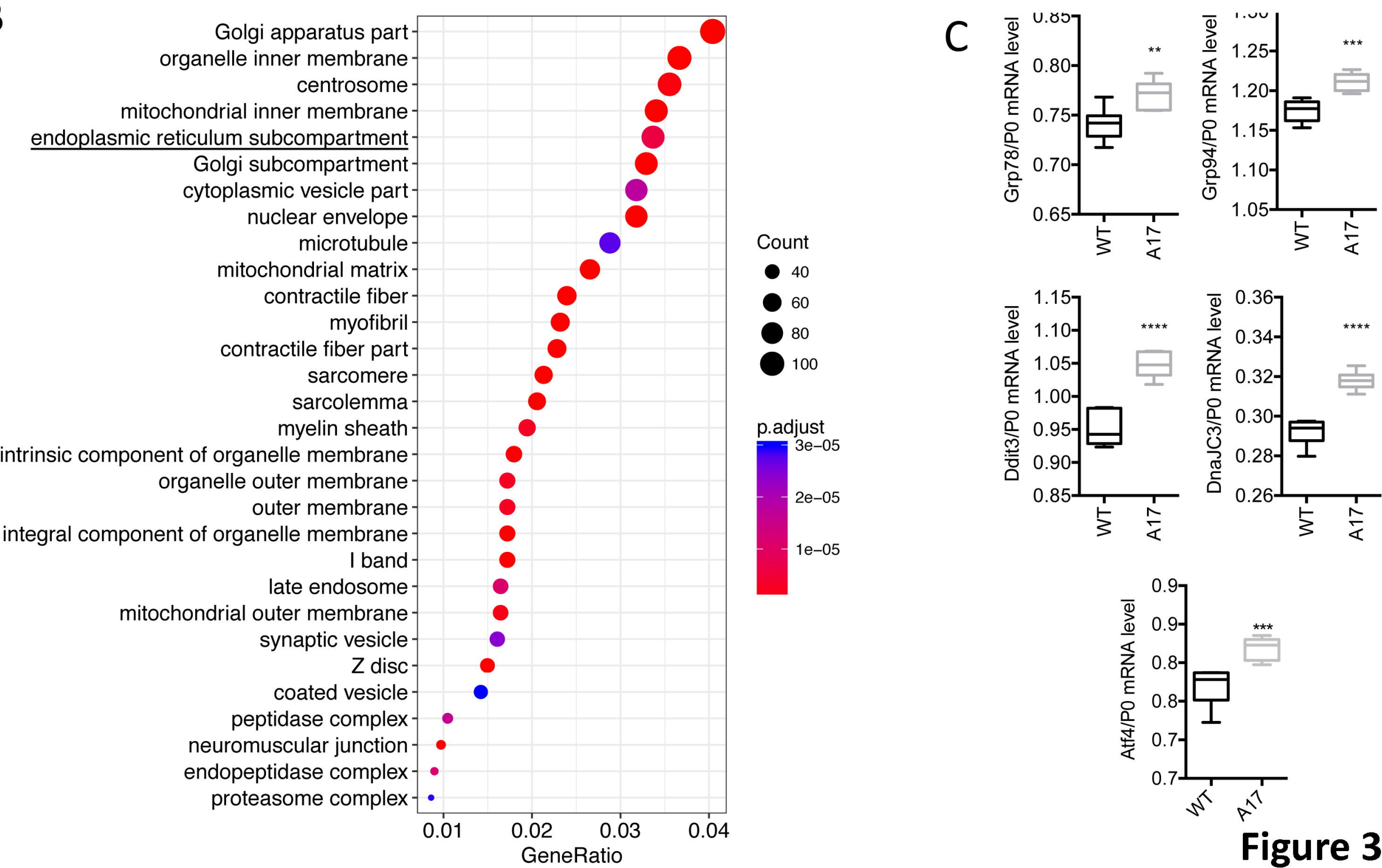

Figure 3 
A

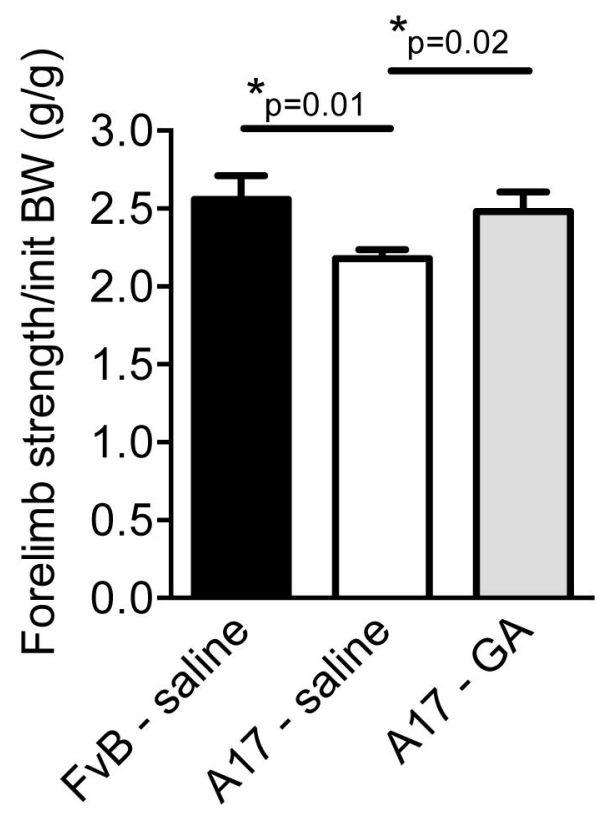

C

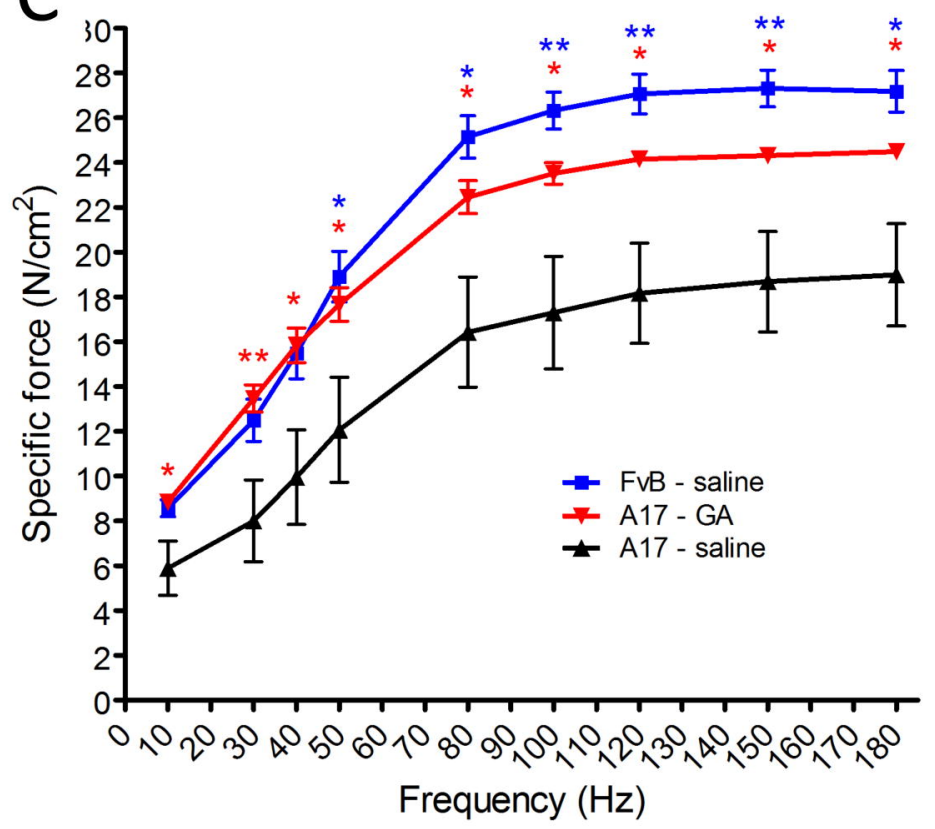

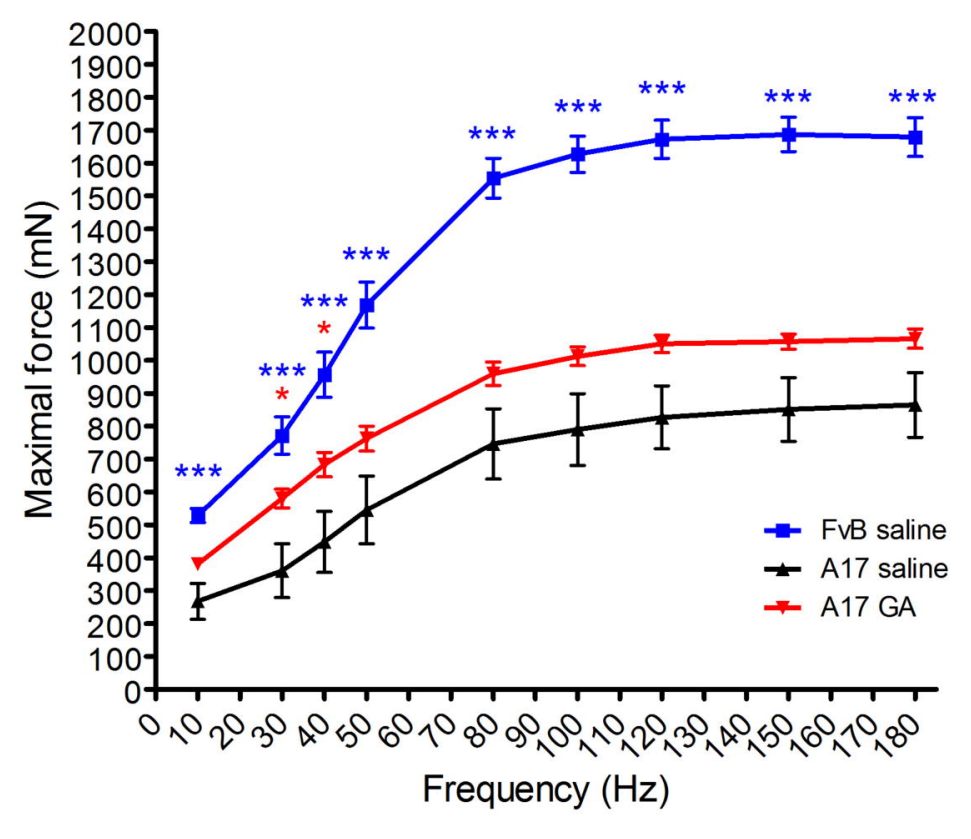

D

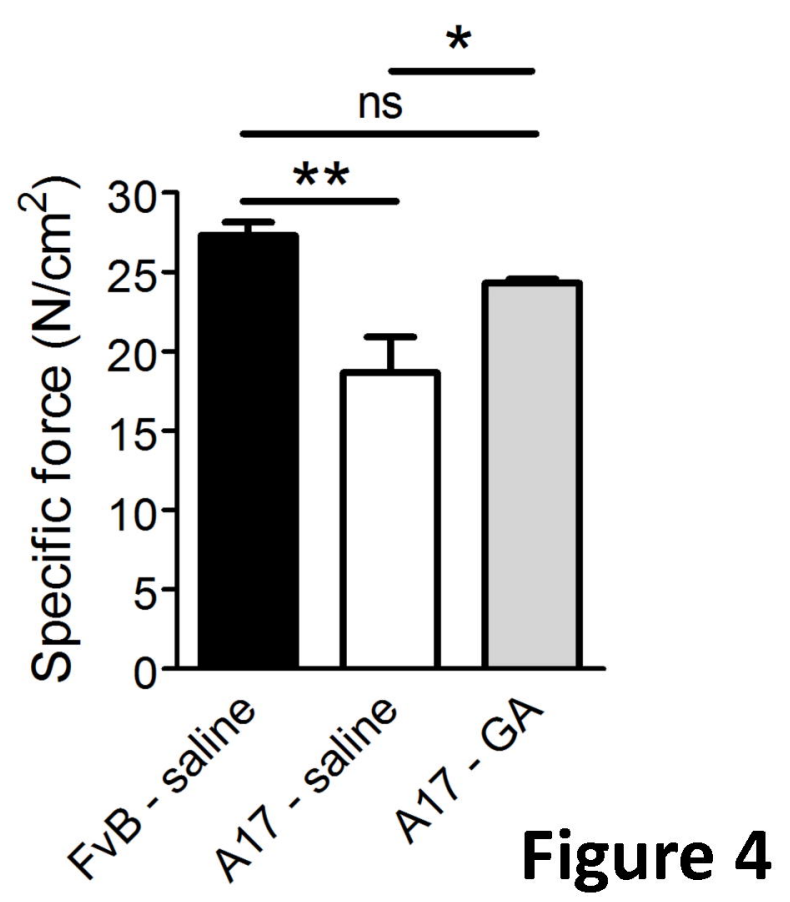


A FvB - saline

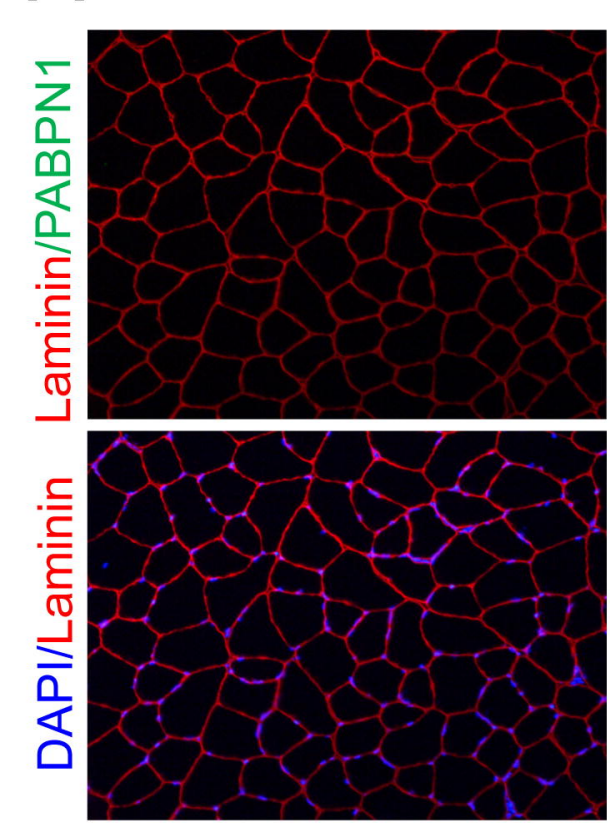

A17 - saline

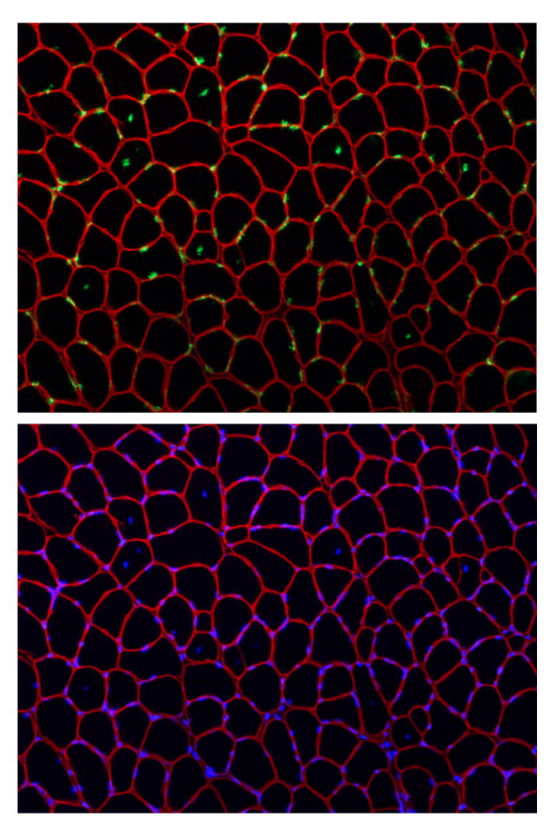

D FvB - saline

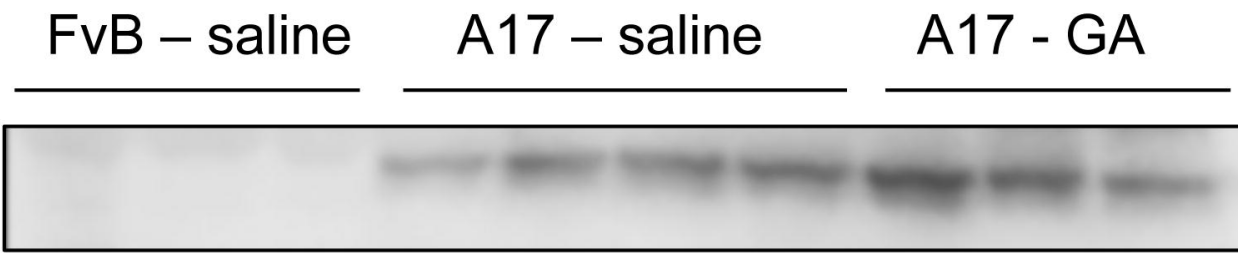

PABPN1

Tubulin
B $\quad C$
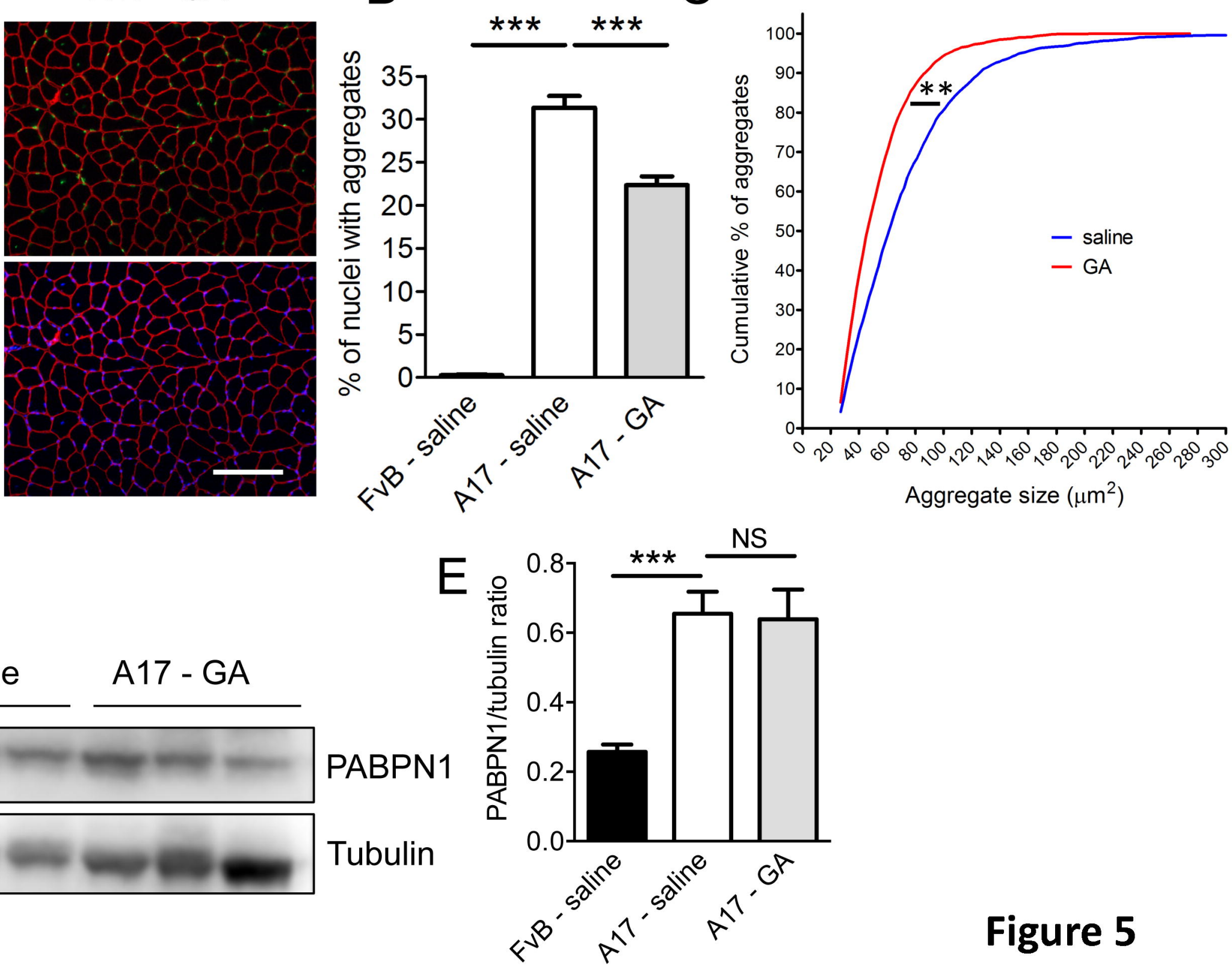
Figure 6

A

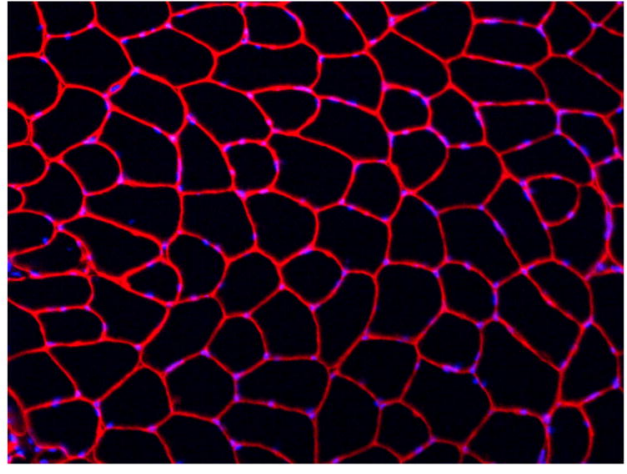

B

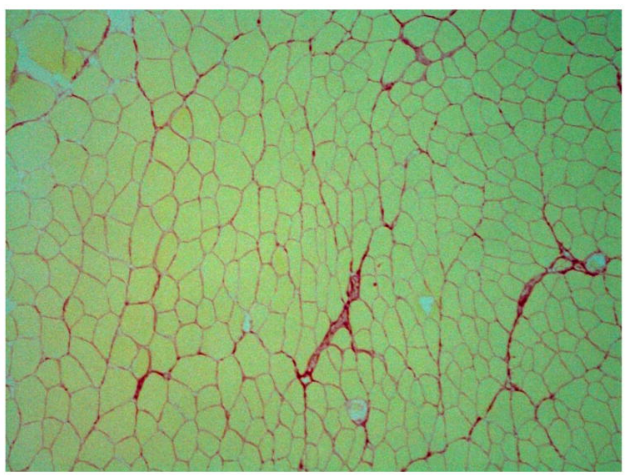

C

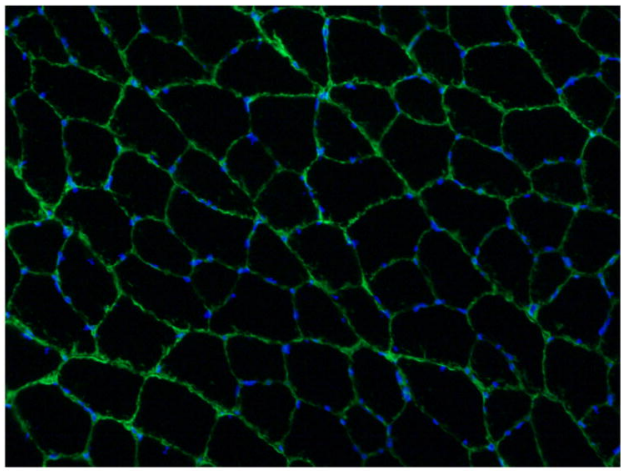

FvB - saline
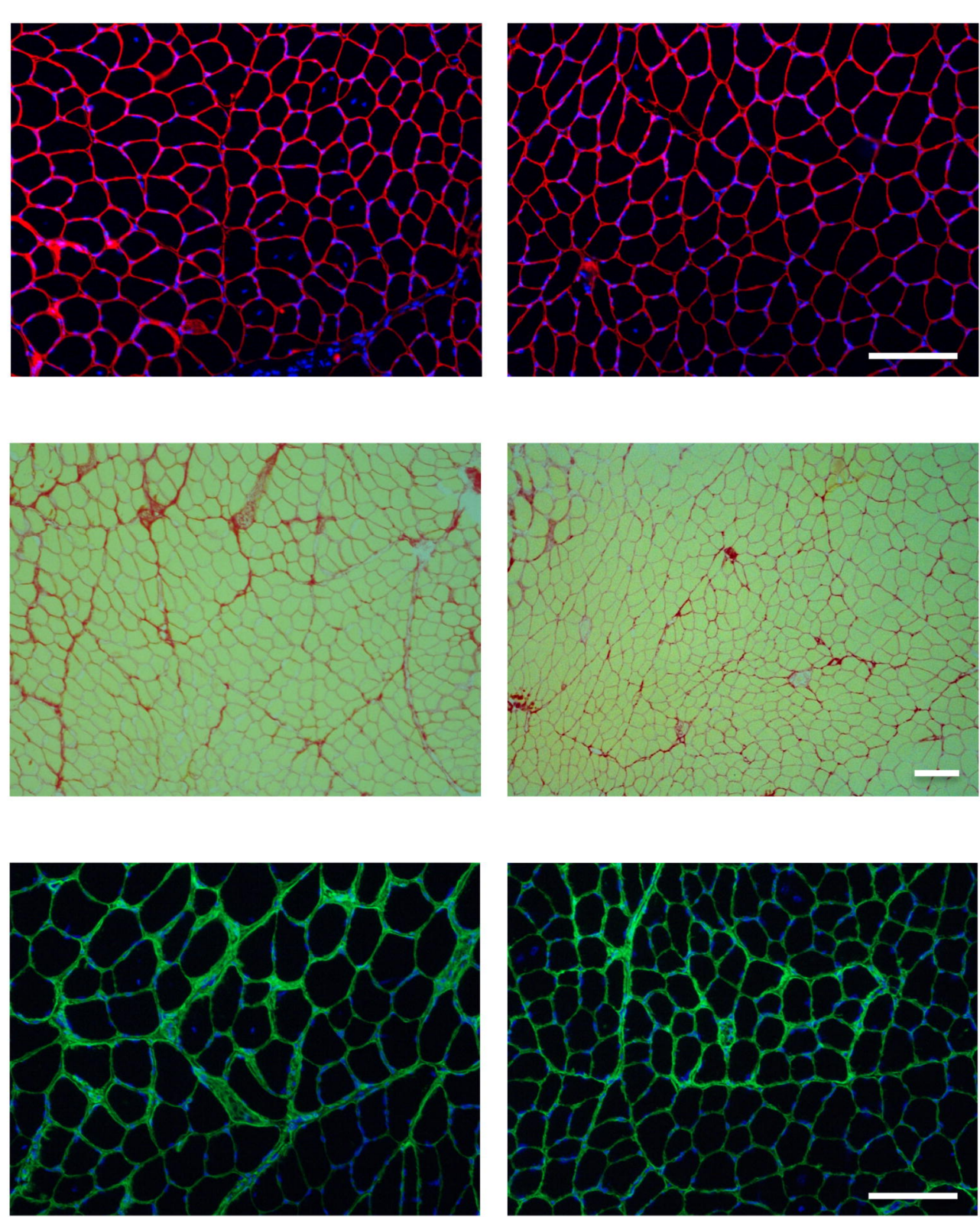

A17 - saline
A17 - GA
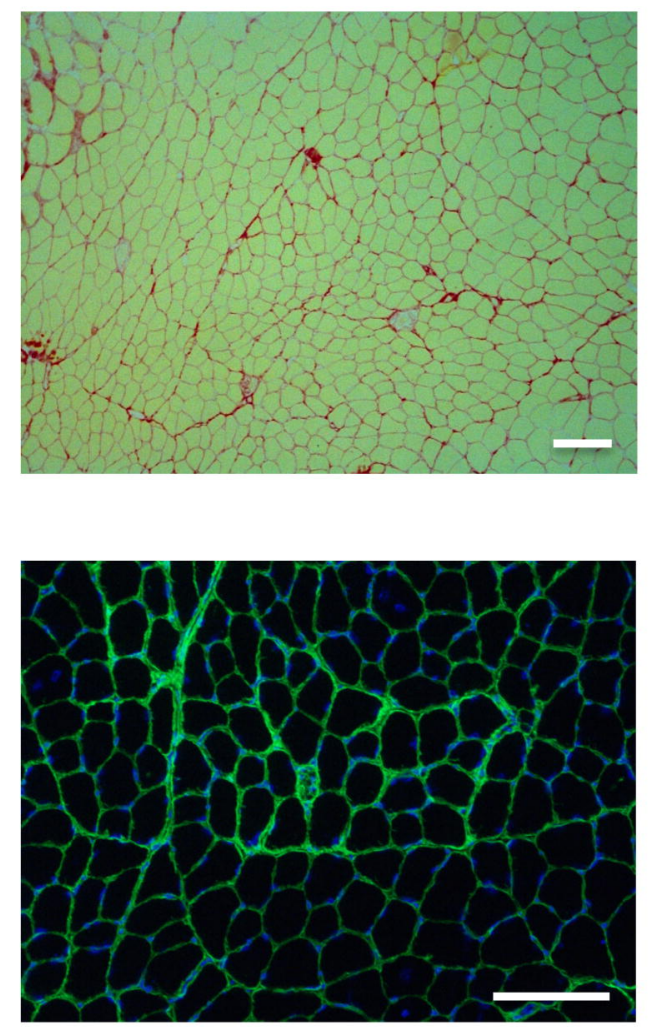

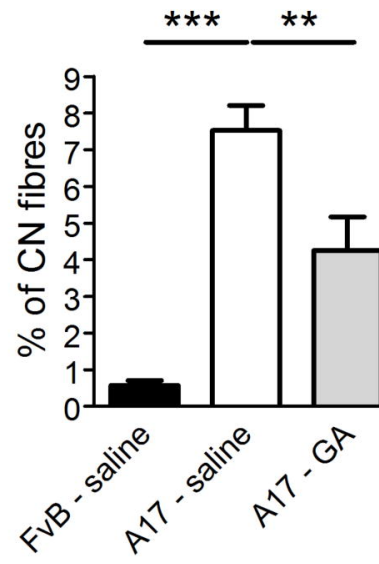

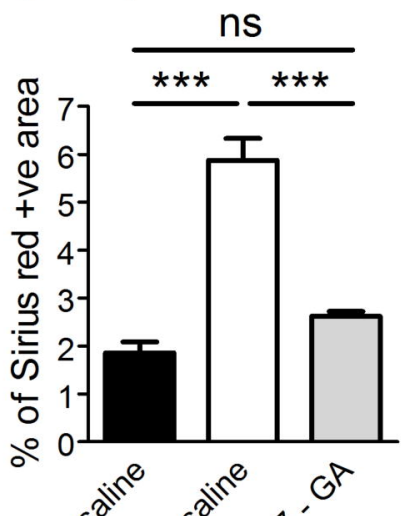

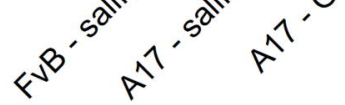

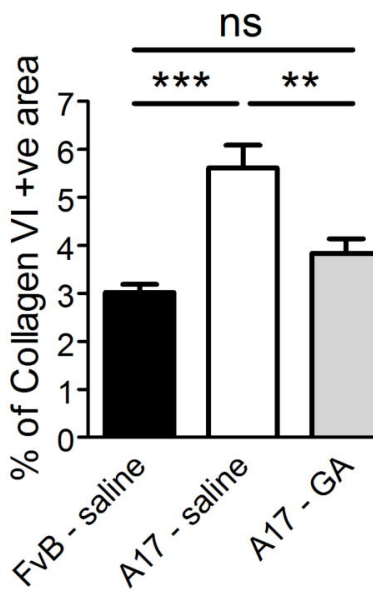


Figure 7

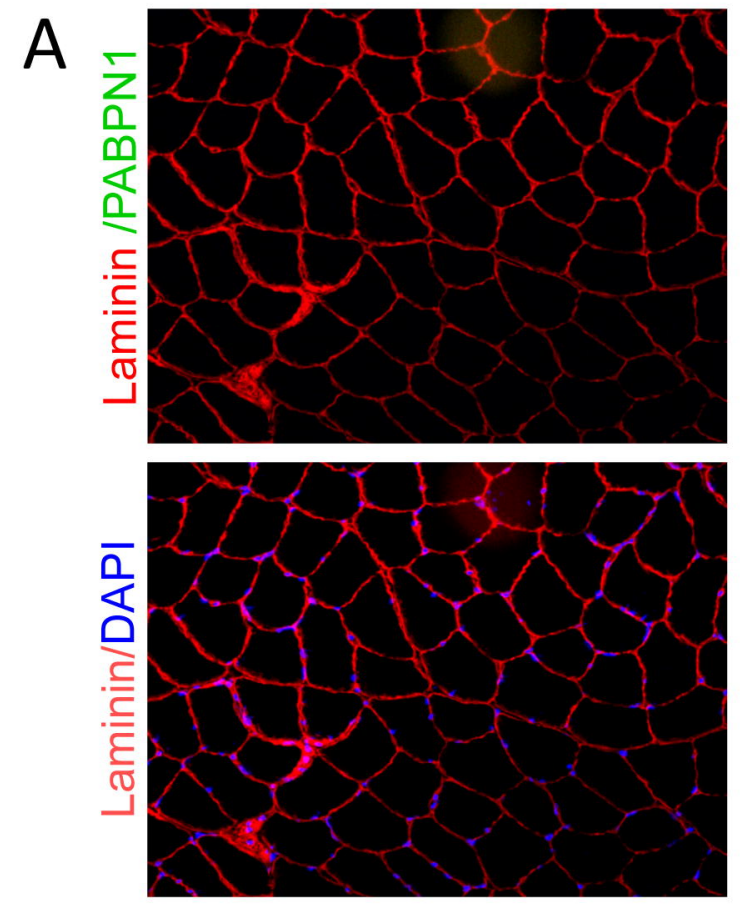

FvB - saline

B

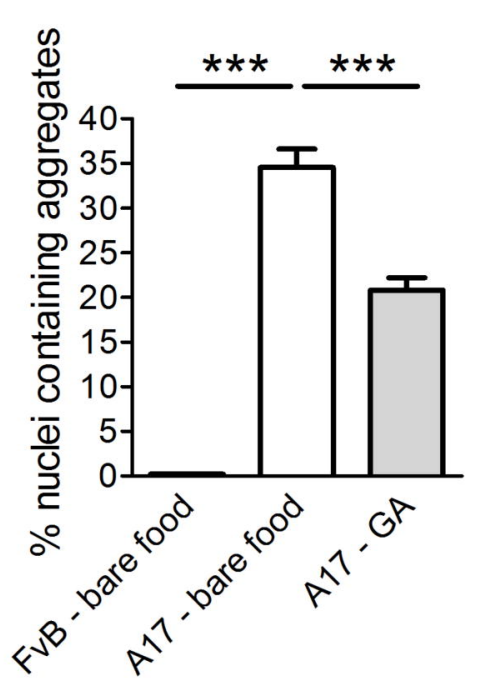

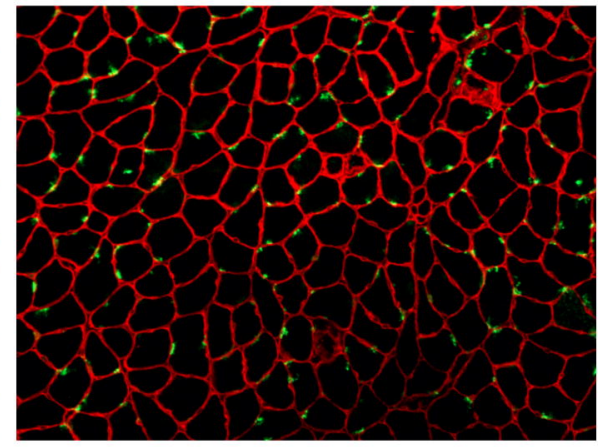

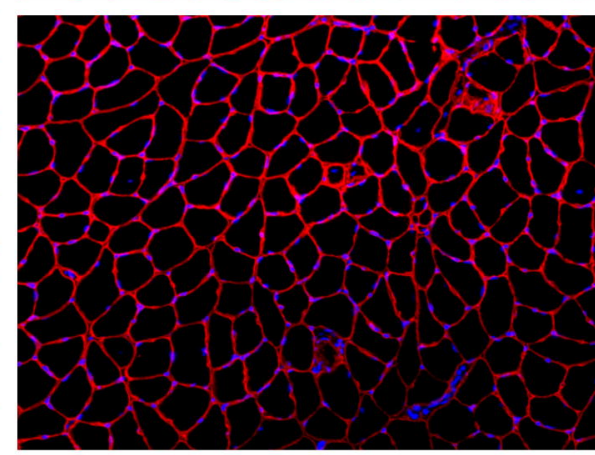

A17 - saline

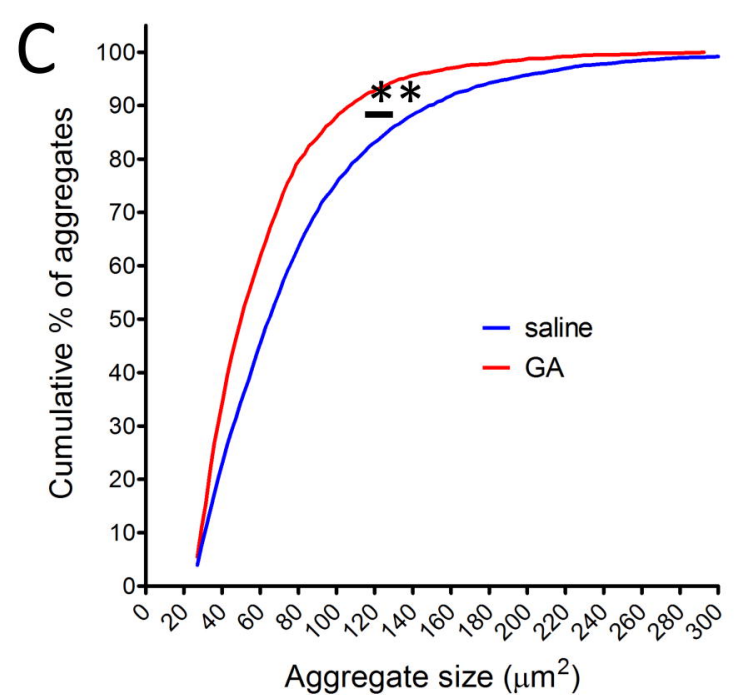

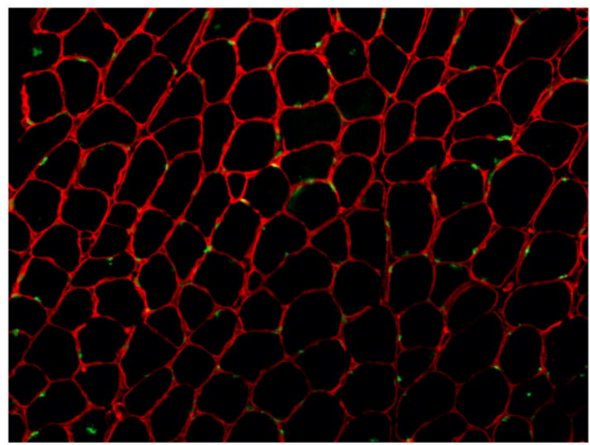

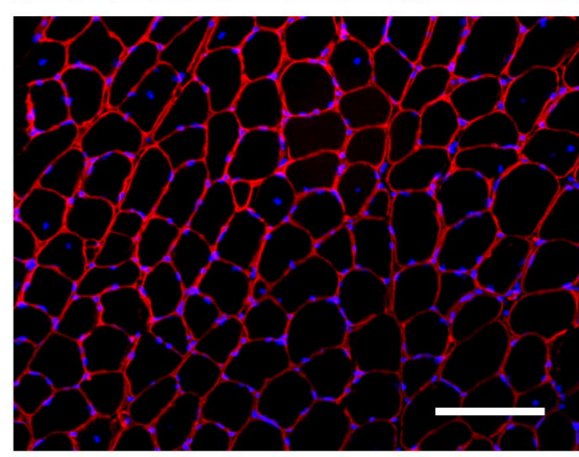

A17 - GA

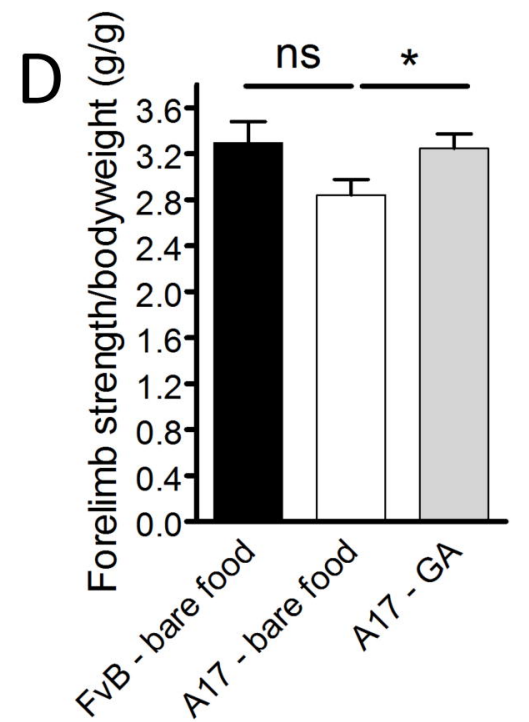

